\title{
An iterative method for approximating the common solutions of a variational inequality, a mixed equilibrium problem and a hierarchical fixed point problem
}

\author{
Abdellah Bnouhachem ${ }^{1,2^{*}}$ and Muhammad Aslam Noor ${ }^{3}$
}

\author{
*Correspondence: \\ babedallah@yahoo.com \\ ${ }^{1}$ School of Management Science \\ and Engineering, Nanjing University, \\ Nanjing, 210093, P.R. China \\ 2ENSA, Ibn Zohr University, Agadir, \\ 1136, Morocco \\ Full list of author information is \\ available at the end of the article
}

\begin{abstract}
In this paper, we suggest and analyze an iterative scheme for finding the approximate element of the common set of solutions of a generalized equilibrium problem, a variational inequality problem and a hierarchical fixed point problem in a real Hilbert space. We also consider the strong convergence of the proposed method under some conditions. Results proved in this paper may be viewed as an improvement and refinement of the previously known results.

MSC: 49J30; 47H09; 47J20
\end{abstract}

Keywords: mixed equilibrium problem; variational inequality problem; hierarchical fixed point problem; projection method; strictly pseudo-contractive mapping

\section{Introduction}

Let $H$ be a real Hilbert space, whose inner product and norm are denoted by $\langle\cdot, \cdot\rangle$ and $\|\cdot\|$. Let $C$ be a nonempty closed convex subset of $H$, and $A$ is a mapping from $C$ into $H$. A classical variational inequality problem, denoted by $\operatorname{VI}(A, C)$, is to find a vector $u \in C$ such that

$$
\langle v-u, A u\rangle \geq 0, \quad \forall v \in C
$$

The solution of $\operatorname{VI}(A, C)$ is denoted by $\Omega^{*}$. It is easy to observe that

$$
u^{*} \in \Omega^{*} \quad \Longleftrightarrow \quad u^{*}=P_{C}\left[u^{*}-\rho A u^{*}\right], \quad \text { where } \rho>0 .
$$

We now have a variety of techniques to suggest and analyze various iterative algorithms for solving variational inequalities and the related optimization problems, see [1-22]. The fixed-point theory has played an important role in the development of various algorithms for solving variational inequalities. Using the projection operator technique, one usually establishes an equivalence between the variational inequalities and the fixed-point problem. This alternative equivalent formulation was used by Lions and Stampacchia [8] to study the existence of a solution of the variational inequalities.

We introduce the following definitions, which are useful in the following analysis.

C2013 Bnouhachem and Noor; licensee Springer. This is an Open Access article distributed under the terms of the Creative Commons Attribution License (http://creativecommons.org/licenses/by/2.0), which permits unrestricted use, distribution, and reproduction in any medium, provided the original work is properly cited. 
Definition 1.1 The mapping $T: C \rightarrow H$ is said to be

(a) monotone if

$$
\langle T x-T y, x-y\rangle \geq 0, \quad \forall x, y \in C
$$

(b) strongly monotone if there exists an $\alpha>0$ such that

$$
\langle T x-T y, x-y\rangle \geq \alpha\|x-y\|^{2}, \quad \forall x, y \in C ;
$$

(c) $\alpha$-inverse strongly monotone if there exists an $\alpha>0$ such that

$$
\langle T x-T y, x-y\rangle \geq \alpha\|T x-T y\|^{2}, \quad \forall x, y \in C ;
$$

(d) nonexpansive if

$$
\|T x-T y\| \leq\|x-y\|, \quad \forall x, y \in C
$$

(e) $k$-Lipschitz continuous if there exists a constant $k>0$ such that

$$
\|T x-T y\| \leq k\|x-y\|, \quad \forall x, y \in C ;
$$

(f) contraction on $C$ if there exists a constant $0 \leq k<1$ such that

$$
\|T x-T y\| \leq k\|x-y\|, \quad \forall x, y \in C .
$$

It is easy to observe that every $\alpha$-inverse strongly monotone $T$ is monotone and Lipschitz continuous. A mapping $T: C \rightarrow H$ is called $k$-strict pseudo-contraction if there exists a constant $0 \leq k<1$ such that

$$
\|T x-T y\|^{2} \leq\|x-y\|^{2}+k\|(I-T) x-(I-T) y\|^{2}, \quad \forall x, y \in C .
$$

The fixed point problem for the mapping $T$ is to find $x \in C$ such that

$$
T x=x .
$$

We denote $F(T)$ the set of solutions of (1.3). It is well known that the class of strict pseudocontractions strictly includes the class of nonexpansive mappings, then $F(T)$ is closed and convex, and $P_{F(T)}$ is well defined (see [22]).

The mixed equilibrium problem, denoted by $M E P$, is to find $x \in C$ such that

$$
F(x, y)+\langle D x, y-x\rangle \geq 0, \quad \forall y \in C,
$$

where $F: C \times C \rightarrow \mathbb{R}$ is a bifunction, and $D: C \rightarrow H$ is a nonlinear mapping. This problem was introduced and studied by Moudafi and Théra [13] and Moudafi [14]. The set of solutions of (1.4) is denoted by

$$
\operatorname{MEP}(F):=\{x \in C: F(x, y)+\langle D x, y-x\rangle \geq 0, \forall y \in C\} .
$$


If $D=0$, then it is reduced to the equilibrium problem, which is to find $x \in C$ such that

$$
F(x, y) \geq 0, \quad \forall y \in C .
$$

The solution set of (1.6) is denoted by $E P(F)$. Numerous problems in physics, optimization, and economics reduce to find a solution of (1.6), see [4, 7, 16, 17]. In 1997, Combettes and Hirstoaga [5] introduced an iterative scheme of finding the best approximation to the initial data when $E P(F)$ is nonempty. Recently Plubtieng and Punpaeng [16] introduced an iterative method for finding the common element of the set $F(T) \cap \Omega^{*} \cap E P(F)$.

Let $S: C \rightarrow H$ be a nonexpansive mapping. The following problem is called a hierarchical fixed point problem: Find $x \in F(T)$ such that

$$
\langle x-S x, y-x\rangle \geq 0, \quad \forall y \in F(T) .
$$

It is known that the hierarchical fixed point problem (1.7) links with some monotone variational inequalities and convex programming problems; see $[6,20]$. Various methods have been proposed to solve the hierarchical fixed point problem; see Moudafi [15], Mainge and Moudafi in [9], Marino and Xu in [11] and Cianciaruso et al. [3]. Very recently, Yao et al. [20] introduced the following strong convergence iterative algorithm to solve problem (1.7):

$$
\begin{aligned}
& y_{n}=\beta_{n} S x_{n}+\left(1-\beta_{n}\right) x_{n}, \\
& x_{n+1}=P_{C}\left[\alpha_{n} f\left(x_{n}\right)+\left(1-\alpha_{n}\right) T y_{n}\right], \quad \forall n \geq 0,
\end{aligned}
$$

where $f: C \rightarrow H$ is a contraction mapping, and $\left\{\alpha_{n}\right\}$ and $\left\{\beta_{n}\right\}$ are two sequences in $(0,1)$. Under some certain restrictions on parameters, Yao et al. proved that the sequence $\left\{x_{n}\right\}$ generated by (1.8) converges strongly to $z \in F(T)$, which is the unique solution of the following variational inequality:

$$
\langle(I-f) z, y-z\rangle \geq 0, \quad \forall y \in F(T) .
$$

By changing the restrictions on parameters, the authors obtained another result on the iterative scheme (1.8), the sequence $\left\{x_{n}\right\}$ generated by (1.8) converges strongly to a point $z \in F(T)$, which is the unique solution of the following variational inequality:

$$
\left\langle\frac{1}{\tau}(I-f) z+(I-S) z, y-z\right\rangle \geq 0, \quad \forall y \in F(T) .
$$

Let $S: C \rightarrow H$ be a nonexpansive mapping, and $\left\{T_{i}\right\}_{i=1}^{\infty}: C \rightarrow C$ is a countable family of nonexpansive mappings. Very recently, Gu et al. [6] introduced the following iterative algorithm:

$$
\begin{aligned}
& y_{n}=P_{C}\left[\beta_{n} S x_{n}+\left(1-\beta_{n}\right) x_{n}\right], \\
& x_{n+1}=P_{C}\left[\alpha_{n} f\left(x_{n}\right)+\sum_{i=1}^{n}\left(\alpha_{i-1}-\alpha_{i}\right) T_{i} y_{n}\right], \quad \forall n \geq 1,
\end{aligned}
$$


where $\alpha_{0}=1,\left\{\alpha_{n}\right\}$ is a strictly decreasing sequence in $(0,1)$, and $\left\{\beta_{n}\right\}$ is a sequence in $(0,1)$. Under some certain conditions on parameters, Gu et al. proved that the sequence $\left\{x_{n}\right\}$ generated by (1.11) converges strongly to $z \in \bigcap_{i=1}^{\infty} F\left(T_{i}\right)$, which is a unique solution of one of the variational inequalities (1.9) and (1.10).

In this paper, motivated by the work of Yao et al. [20] and Gu et al. [6] and by the recent work going in this direction, we give an iterative method for finding the approximate element of the common set of solutions of (1.1), (1.4) and (1.7) for a strictly pseudocontraction mapping in a real Hilbert space. We establish a strong convergence theorem based on this method. The presented method improves and generalizes many known results for solving equilibrium problems, variational inequality problems and hierarchical fixed point problems, see, e.g., $[3,6,9,20]$ and relevant references cited therein.

\section{Preliminaries}

In this section, we list some fundamental lemmas that are useful in the consequent analysis. The first lemma provides some basic properties of projection onto $C$.

Lemma 2.1 Let $P_{C}$ denote the projection of $H$ onto $C$. Then we have the following inequalities:

$$
\begin{aligned}
& \left\langle z-P_{C}[z], P_{C}[z]-v\right\rangle \geq 0, \quad \forall z \in H, v \in C ; \\
& \left\langle u-v, P_{C}[u]-P_{C}[v]\right\rangle \geq\left\|P_{C}[u]-P_{C}[v]\right\|^{2}, \quad \forall u, v \in H ; \\
& \left\|P_{C}[u]-P_{C}[v]\right\| \leq\|u-v\|, \quad \forall u, v \in H ; \\
& \left\|u-P_{C}[z]\right\|^{2} \leq\|z-u\|^{2}-\left\|z-P_{C}[z]\right\|^{2}, \quad \forall z \in H, u \in C .
\end{aligned}
$$

Lemma 2.2 [2] Let $F: C \times C \rightarrow \mathbb{R}$ be a bifunction satisfying the following assumptions:

(i) $F(x, x)=0, \forall x \in C$;

(ii) $F$ is monotone, i.e., $F(x, y)+F(y, x) \leq 0, \forall x, y \in C$;

(iii) For each $x, y, z \in C, \lim _{t \rightarrow 0} F(t z+(1-t) x, y) \leq F(x, y)$;

(iv) For each $x \in C, y \rightarrow F(x, y)$ is convex and lower semicontinuous.

Let $r>0$ and $x \in H$. Then there exists $z \in C$ such that

$$
F(z, y)+\frac{1}{r}\langle y-z, z-x\rangle \geq 0, \quad \forall y \in C .
$$

Lemma 2.3 [5] Assume that $F: C \times C \rightarrow \mathbb{R}$ satisfies assumptions (i)-(iv) of Lemma 2.2. For $r>0$ and $\forall x \in H$, define a mapping $T_{r}: H \rightarrow C$ as follows:

$$
T_{r}(x)=\left\{z \in C: F(z, y)+\frac{1}{r}\langle y-z, z-x\rangle \geq 0, \forall y \in C\right\} .
$$

Then the following hold:

(i) $T_{r}$ is single-valued;

(ii) $T_{r}$ is firmly nonexpansive, i.e.,

$$
\left\|T_{r} x-T_{r} y\right\|^{2} \leq\left\langle T_{r} x-T_{r} y, x-y\right\rangle, \quad \forall x, y \in H
$$

(iii) $F\left(T_{r}\right)=E P(F)$;

(iv) $E P(F)$ is closed and convex. 
Lemma 2.4 [21] Let $C$ be a nonempty closed convex subset of a real Hilbert space $H$. If $T: C \rightarrow C$ is a $k$-strict pseudo-contraction, then

(i) The mapping $I-T$ is demiclosed at 0 , i.e., if $\left\{x_{n}\right\}$ is a sequence in $C$ weakly converging to $x$, and if $\left\{(I-T) x_{n}\right\}$ converges strongly to 0 , then $(I-T) x=0$;

(ii) The set $F(T)$ of $T$ is closed and convex, so that the projection $P_{F(T)}$ is well defined.

Lemma 2.5 [10] Let $H$ be a real Hilbert space. Then the following inequality holds:

$$
\|x+y\|^{2} \leq\|x\|^{2}+2\langle y, x+y\rangle, \quad \forall x, y \in H .
$$

Lemma 2.6 [19] Assume that $\left\{a_{n}\right\}$ is a sequence of nonnegative real numbers such that

$$
a_{n+1} \leq\left(1-\gamma_{n}\right) a_{n}+\delta_{n}
$$

where $\left\{\gamma_{n}\right\}$ is a sequence in $(0,1)$, and $\delta_{n}$ is a sequence such that

(1) $\sum_{n=1}^{\infty} \gamma_{n}=\infty$;

(2) $\lim \sup _{n \rightarrow \infty} \delta_{n} / \gamma_{n} \leq 0$ or $\sum_{n=1}^{\infty}\left|\delta_{n}\right|<\infty$

Then $\lim _{n \rightarrow \infty} a_{n}=0$.

Lemma 2.7 [1] Let C be a closed convex subset of H. Let $\left\{x_{n}\right\}$ be a bounded sequence in $H$. Assume that

(i) The weak $w$-limit set $w_{w}\left(x_{n}\right) \subset C$, where $w_{w}\left(x_{n}\right)=\left\{x: x_{n_{i}} \rightarrow x\right\}$.

(ii) For each $z \in C, \lim _{n \rightarrow \infty}\left\|x_{n}-z\right\|$ exists.

Then $\left\{x_{n}\right\}$ is weakly convergent to a point in $C$.

Lemma 2.8 [22] Let $H$ be a Hilbert space, $C$ be a closed and convex subset of $H$, and $T: C \rightarrow C$ be a $k$-strict pseudo-contraction mapping. Define a mapping $V: C \rightarrow H$ by $V x=\lambda x+(1-\lambda) T x, \forall x \in C$. Then, as $k \leq \lambda<1, V$ is a nonexpansive mapping such that $F(V)=F(T)$.

Lemma 2.9 [6] Let $H$ be a Hilbert space, $C$ be a closed and convex subset of $H$, and $T$ : $C \rightarrow C$ be a nonexpansive mapping such that $F(T) \neq \emptyset$. Then

$$
\|T x-x\|^{2} \leq 2\left\langle x-T x, x-x^{\prime}\right\rangle, \quad \forall x^{\prime} \in F(T), \forall x \in C .
$$

\section{The proposed method and some properties}

In this section, we suggest and analyze our method for finding the common solutions of the variational inequality (1.1), the mixed equilibrium problem (1.4) and the hierarchical fixed point problem (1.7).

Let $C$ be a nonempty closed convex subset of a real Hilbert space $H$. Let $D, A: C \rightarrow H$ be $\theta, \alpha$-inverse strongly monotone mappings, respectively. Let $F: C \times C \rightarrow \mathbb{R}$ be a bifunction satisfying assumptions (i)-(iv) of Lemma 2.2, $S: C \rightarrow H$ be a nonexpansive mapping and $\left\{T_{i}\right\}_{i=1}^{\infty}: C \rightarrow C$ is a countable family of $k_{i}$-strict pseudo-contraction mappings such that $F(T) \cap \Omega^{*} \cap M E P(F) \neq \emptyset$, where $F(T)=\bigcap_{i=1}^{\infty} F\left(T_{i}\right)$. Let $f$ be a $\rho$-contraction mapping. 
Algorithm 3.1 For a given $x_{0} \in C$ arbitrarily, let the iterative sequences $\left\{u_{n}\right\},\left\{x_{n}\right\},\left\{y_{n}\right\}$ and $\left\{z_{n}\right\}$ be generated by

$$
\begin{aligned}
& F\left(u_{n}, y\right)+\left\langle D x_{n}, y-u_{n}\right\rangle+\frac{1}{r_{n}}\left\langle y-u_{n}, u_{n}-x_{n}\right\rangle \geq 0, \quad \forall y \in C ; \\
& z_{n}=P_{C}\left[u_{n}-\lambda_{n} A u_{n}\right] ; \\
& y_{n}=P_{C}\left[\beta_{n} S x_{n}+\left(1-\beta_{n}\right) z_{n}\right] ; \\
& x_{n+1}=P_{C}\left[\alpha_{n} f\left(x_{n}\right)+\sum_{i=1}^{n}\left(\alpha_{i-1}-\alpha_{i}\right) V_{i} y_{n}\right], \quad \forall n \geq 0,
\end{aligned}
$$

where $V_{i}=k_{i} I+\left(1-k_{i}\right) T_{i}, 0 \leq k_{i}<1,\left\{\lambda_{n}\right\} \subset(0,2 \alpha),\left\{r_{n}\right\} \subset(0,2 \theta], \alpha_{0}=1,\left\{\alpha_{n}\right\}$ is a strictly decreasing sequence in $(0,1)$, and $\left\{\beta_{n}\right\}$ is a sequence in $(0,1)$ satisfying the following conditions:

(a) $\lim _{n \rightarrow \infty} \alpha_{n}=0$ and $\sum_{n=1}^{\infty} \alpha_{n}=\infty$,

(b) $\lim _{n \rightarrow \infty}\left(\beta_{n} / \alpha_{n}\right)=0$,

(c) $\sum_{n=1}^{\infty}\left|\alpha_{n-1}-\alpha_{n}\right|<\infty$ and $\sum_{n=1}^{\infty}\left|\beta_{n-1}-\beta_{n}\right|<\infty$,

(d) $\liminf _{n \rightarrow \infty} r_{n}>0$ and $\sum_{n=1}^{\infty}\left|r_{n-1}-r_{n}\right|<\infty$,

(e) $\liminf _{n \rightarrow \infty} \lambda_{n}<\limsup \sup _{n \rightarrow \infty} \lambda_{n}<2 \alpha$ and $\sum_{n=1}^{\infty}\left|\lambda_{n-1}-\lambda_{n}\right|<\infty$.

Remark 3.1 It is easy to verify that Algorithm 3.1 includes some existing methods (e.g., [3, $6,9,20])$ as special cases. Therefore, the new algorithm is expected to be widely applicable.

Lemma 3.1 Let $x^{*} \in F(T) \cap \Omega^{*} \cap \operatorname{MEP}(F)$. Then $\left\{x_{n}\right\},\left\{u_{n}\right\},\left\{z_{n}\right\}$ and $\left\{y_{n}\right\}$ are bounded.

Proof First, we show that the mapping $\left(I-r_{n} D\right)$ is nonexpansive. For any $x, y \in C$,

$$
\begin{aligned}
\left\|\left(I-r_{n} D\right) x-\left(I-r_{n} D\right) y\right\|^{2} \\
\quad=\left\|(x-y)-r_{n}(D x-D y)\right\|^{2} \\
\quad=\|x-y\|^{2}-2 r_{n}\langle x-y, D x-D y\rangle+r_{n}^{2}\|D x-D y\|^{2} \\
\quad \leq\|x-y\|^{2}-r_{n}\left(2 \theta-r_{n}\right)\|D x-D y\|^{2} \\
\quad \leq\|x-y\|^{2} .
\end{aligned}
$$

Similarly, we can show that the mapping $\left(I-\lambda_{n} A\right)$ is nonexpansive. It follows from Lemma 2.3 that $u_{n}=T_{r_{n}}\left(x_{n}-r_{n} D x_{n}\right)$. Let $x^{*} \in F(T) \cap \Omega^{*} \cap M E P(F)$, we have $x^{*}=T_{r_{n}}\left(x^{*}-\right.$ $\left.r_{n} D x^{*}\right)$.

$$
\begin{aligned}
& \left\|u_{n}-x^{*}\right\|^{2} \\
& \quad=\left\|T_{r_{n}}\left(x_{n}-r_{n} D x_{n}\right)-T_{r_{n}}\left(x^{*}-r_{n} D x^{*}\right)\right\|^{2} \\
& \quad \leq\left\|\left(x_{n}-r_{n} D x_{n}\right)-\left(x^{*}-r_{n} D x^{*}\right)\right\|^{2} \\
& \quad \leq\left\|x_{n}-x^{*}\right\|^{2}-r_{n}\left(2 \theta-r_{n}\right)\left\|D x_{n}-D x^{*}\right\|^{2} \\
& \quad \leq\left\|x_{n}-x^{*}\right\|^{2} .
\end{aligned}
$$


Since the mapping $A$ is $\alpha$-inverse strongly monotone, we have

$$
\begin{aligned}
\left\|z_{n}-x^{*}\right\|^{2} & =\left\|P_{C}\left[u_{n}-\lambda_{n} A u_{n}\right)-P_{C}\left[x^{*}-\lambda_{n} A x^{*}\right]\right\|^{2} \\
& \leq\left\|u_{n}-x^{*}-\lambda_{n}\left(A u_{n}-A x^{*}\right)\right\|^{2} \\
& \leq\left\|u_{n}-x^{*}\right\|^{2}-\lambda_{n}\left(2 \alpha-\lambda_{n}\right)\left\|A u_{n}-A x^{*}\right\|^{2} \\
& \leq\left\|u_{n}-x^{*}\right\|^{2} \\
& \leq\left\|x_{n}-x^{*}\right\|^{2} .
\end{aligned}
$$

Next, we prove that the sequence $\left\{x_{n}\right\}$ is bounded, without loss of generality, we can assume that $\beta_{n} \leq \alpha_{n}$ for all $n \geq 1$. From (3.1), we have

$$
\begin{aligned}
& \left\|x_{n+1}-x^{*}\right\| \leq\left\|\alpha_{n} f\left(x_{n}\right)+\sum_{i=1}^{n}\left(\alpha_{i-1}-\alpha_{i}\right) V_{i} y_{n}-\alpha_{n} x^{*}-\sum_{i=1}^{n}\left(\alpha_{i-1}-\alpha_{i}\right) V_{i} x^{*}\right\| \\
& \leq \alpha_{n}\left\|f\left(x_{n}\right)-f\left(x^{*}\right)\right\|+\alpha_{n}\left\|f\left(x^{*}\right)-x^{*}\right\|+\sum_{i=1}^{n}\left(\alpha_{i-1}-\alpha_{i}\right)\left\|V_{i} y_{n}-V_{i} x^{*}\right\| \\
& \leq \alpha_{n}\left\|f\left(x_{n}\right)-f\left(x^{*}\right)\right\|+\alpha_{n}\left\|f\left(x^{*}\right)-x^{*}\right\|+\sum_{i=1}^{n}\left(\alpha_{i-1}-\alpha_{i}\right)\left\|y_{n}-x^{*}\right\| \\
& =\alpha_{n}\left\|f\left(x_{n}\right)-f\left(x^{*}\right)\right\|+\alpha_{n}\left\|f\left(x^{*}\right)-x^{*}\right\|+\left(1-\alpha_{n}\right)\left\|\beta_{n} S x_{n}+\left(1-\beta_{n}\right) z_{n}-x^{*}\right\| \\
& \leq \alpha_{n}\left\|f\left(x_{n}\right)-f\left(x^{*}\right)\right\|+\alpha_{n}\left\|f\left(x^{*}\right)-x^{*}\right\| \\
& +\left(1-\alpha_{n}\right)\left(\beta_{n}\left\|S x_{n}-S x^{*}\right\|+\beta_{n}\left\|S x^{*}-x^{*}\right\|+\left(1-\beta_{n}\right)\left\|z_{n}-x^{*}\right\|\right) \\
& \leq \alpha_{n} \rho\left\|x_{n}-x^{*}\right\|+\alpha_{n}\left\|f\left(x^{*}\right)-x^{*}\right\|+\left(1-\alpha_{n}\right)\left(\beta_{n}\left\|x_{n}-x^{*}\right\|+\beta_{n}\left\|S x^{*}-x^{*}\right\|\right. \\
& \left.+\left(1-\beta_{n}\right)\left\|x_{n}-x^{*}\right\|\right) \\
& =\left(1-\alpha_{n}(1-\rho)\right)\left\|x_{n}-x^{*}\right\|+\alpha_{n}\left\|f\left(x^{*}\right)-x^{*}\right\|+\left(1-\alpha_{n}\right) \beta_{n}\left\|S x^{*}-x^{*}\right\| \\
& \leq\left(1-\alpha_{n}(1-\rho)\right)\left\|x_{n}-x^{*}\right\|+\alpha_{n}\left\|f\left(x^{*}\right)-x^{*}\right\|+\beta_{n}\left\|S x^{*}-x^{*}\right\| \\
& \leq\left(1-\alpha_{n}(1-\rho)\right)\left\|x_{n}-x^{*}\right\|+\alpha_{n}\left(\left\|f\left(x^{*}\right)-x^{*}\right\|+\left\|S x^{*}-x^{*}\right\|\right) \\
& =\left(1-\alpha_{n}(1-\rho)\right)\left\|x_{n}-x^{*}\right\|+\frac{\alpha_{n}(1-\rho)}{1-\rho}\left(\left\|f\left(x^{*}\right)-x^{*}\right\|+\left\|S x^{*}-x^{*}\right\|\right) \\
& \leq \max \left\{\left\|x_{n}-x^{*}\right\|, \frac{1}{1-\rho}\left(\left\|f\left(x^{*}\right)-x^{*}\right\|+\left\|S x^{*}-x^{*}\right\|\right)\right\} .
\end{aligned}
$$

By induction on $n$, we obtain $\left\|x_{n}-x^{*}\right\| \leq \max \left\{\left\|x_{0}-x^{*}\right\|, \frac{1}{1-\rho}\left(\left\|f\left(x^{*}\right)-x^{*}\right\|+\left\|S x^{*}-x^{*}\right\|\right)\right\}$ for $n \geq 0$ and $x_{0} \in C$. Hence, $\left\{x_{n}\right\}$ is bounded and consequently, we deduce that $\left\{u_{n}\right\},\left\{z_{n}\right\}$ and $\left\{y_{n}\right\}$ are bounded.

Lemma 3.2 Let $x^{*} \in F(T) \cap \Omega^{*} \cap M E P(F)$ and $\left\{x_{n}\right\}$ be the sequence generated by Algorithm 3.1. Then we have

(a) $\lim _{n \rightarrow \infty}\left\|x_{n+1}-x_{n}\right\|=0$.

(b) The weak $w$-limit set $w_{w}\left(x_{n}\right) \subset F(T),\left(w_{w}\left(x_{n}\right)=\left\{x: x_{n_{i}} \rightarrow x\right\}\right)$. 
Proof From the nonexpansivity of the mapping $\left(I-\lambda_{n} A\right)$ and $P_{C}$, we have

$$
\begin{aligned}
\left\|z_{n}-z_{n-1}\right\| & \leq\left\|\left(u_{n}-\lambda_{n} A u_{n}\right)-\left(u_{n-1}-\lambda_{n-1} A u_{n-1}\right)\right\| \\
& =\left\|\left(u_{n}-u_{n-1}\right)-\lambda_{n}\left(A u_{n}-A u_{n-1}\right)-\left(\lambda_{n}-\lambda_{n-1}\right) A u_{n-1}\right\| \\
& \leq\left\|\left(u_{n}-u_{n-1}\right)-\lambda_{n}\left(A u_{n}-A u_{n-1}\right)\right\|+\left|\lambda_{n}-\lambda_{n-1}\right|\left\|A u_{n-1}\right\| \\
& \leq\left\|u_{n}-u_{n-1}\right\|+\left|\lambda_{n}-\lambda_{n-1}\right|\left\|A u_{n-1}\right\| .
\end{aligned}
$$

Next, we estimate that

$$
\begin{aligned}
\left\|y_{n}-y_{n-1}\right\| \leq & \left\|\beta_{n} S x_{n}+\left(1-\beta_{n}\right) z_{n}-\left(\beta_{n-1} S x_{n-1}+\left(1-\beta_{n-1}\right) z_{n-1}\right)\right\| \\
= & \| \beta_{n}\left(S x_{n}-S x_{n-1}\right)+\left(\beta_{n}-\beta_{n-1}\right) S x_{n-1} \\
& +\left(1-\beta_{n}\right)\left(z_{n}-z_{n-1}\right)+\left(\beta_{n-1}-\beta_{n}\right) z_{n-1} \| \\
\leq & \beta_{n}\left\|x_{n}-x_{n-1}\right\|+\left(1-\beta_{n}\right)\left\|z_{n}-z_{n-1}\right\| \\
& +\left|\beta_{n}-\beta_{n-1}\right|\left(\left\|S x_{n-1}\right\|+\left\|z_{n-1}\right\|\right) .
\end{aligned}
$$

It follows from (3.5) and (3.6) that

$$
\begin{aligned}
& \left\|y_{n}-y_{n-1}\right\| \\
& \leq \beta_{n}\left\|x_{n}-x_{n-1}\right\|+\left(1-\beta_{n}\right)\left\{\left\|u_{n}-u_{n-1}\right\|+\left|\lambda_{n}-\lambda_{n-1}\right|\left\|A u_{n-1}\right\|\right\} \\
& \quad+\left|\beta_{n}-\beta_{n-1}\right|\left(\left\|S x_{n-1}\right\|+\left\|z_{n-1}\right\|\right) .
\end{aligned}
$$

On the other hand, $u_{n}=T_{r_{n}}\left(x_{n}-r_{n} D x_{n}\right)$ and $u_{n-1}=T_{r_{n-1}}\left(x_{n-1}-r_{n-1} D x_{n-1}\right)$, we have

$$
F\left(u_{n}, y\right)+\left\langle D x_{n}, y-u_{n}\right\rangle+\frac{1}{r_{n}}\left\langle y-u_{n}, u_{n}-x_{n}\right\rangle \geq 0, \quad \forall y \in C
$$

and

$$
F\left(u_{n-1}, y\right)+\left\langle D x_{n-1}, y-u_{n-1}\right\rangle+\frac{1}{r_{n-1}}\left\langle y-u_{n-1}, u_{n-1}-x_{n-1}\right\rangle \geq 0, \quad \forall y \in C .
$$

Take $y=u_{n-1}$ in (3.8) and $y=u_{n}$ in (3.9), we get

$$
F\left(u_{n}, u_{n-1}\right)+\left\langle D x_{n}, u_{n-1}-u_{n}\right\rangle+\frac{1}{r_{n}}\left\langle u_{n-1}-u_{n}, u_{n}-x_{n}\right\rangle \geq 0
$$

and

$$
F\left(u_{n-1}, u_{n}\right)+\left\langle D x_{n-1}, u_{n}-u_{n-1}\right\rangle+\frac{1}{r_{n-1}}\left\langle u_{n}-u_{n-1}, u_{n-1}-x_{n-1}\right\rangle \geq 0 .
$$

Adding (3.10) and (3.11) and using the monotonicity of $F$, we have

$$
\left\langle D x_{n-1}-D x_{n}, u_{n}-u_{n-1}\right\rangle+\left\langle u_{n}-u_{n-1}, \frac{u_{n-1}-x_{n-1}}{r_{n-1}}-\frac{u_{n}-x_{n}}{r_{n}}\right\rangle \geq 0,
$$


which implies that

$$
\begin{aligned}
0 \leq & \left\langle u_{n}-u_{n-1}, r_{n}\left(D x_{n-1}-D x_{n}\right)+\frac{r_{n}}{r_{n-1}}\left(u_{n-1}-x_{n-1}\right)-\left(u_{n}-x_{n}\right)\right\rangle \\
= & \left\langle u_{n-1}-u_{n}, u_{n}-u_{n-1}+\left(1-\frac{r_{n}}{r_{n-1}}\right) u_{n-1}\right. \\
& \left.+\left(x_{n-1}-r_{n} D x_{n-1}\right)-\left(x_{n}-r_{n} D x_{n}\right)-x_{n-1}+\frac{r_{n}}{r_{n-1}} x_{n-1}\right\rangle \\
= & \left\langle u_{n-1}-u_{n},\left(1-\frac{r_{n}}{r_{n-1}}\right) u_{n-1}+\left(x_{n-1}-r_{n} D x_{n-1}\right)-\left(x_{n}-r_{n} D x_{n}\right)-x_{n-1}+\frac{r_{n}}{r_{n-1}} x_{n-1}\right\rangle \\
& -\left\|u_{n}-u_{n-1}\right\|^{2} \\
= & \left\langle u_{n-1}-u_{n},\left(1-\frac{r_{n}}{r_{n-1}}\right)\left(u_{n-1}-x_{n-1}\right)+\left(x_{n-1}-r_{n} D x_{n-1}\right)-\left(x_{n}-r_{n} D x_{n}\right)\right\rangle \\
& -\left\|u_{n}-u_{n-1}\right\|^{2} \\
\leq & \left\|u_{n-1}-u_{n}\right\|\left\{\left|1-\frac{r_{n}}{r_{n-1}}\right|\left\|u_{n-1}-x_{n-1}\right\|+\left\|\left(x_{n-1}-r_{n} D x_{n-1}\right)-\left(x_{n}-r_{n} D x_{n}\right)\right\|\right\} \\
& -\left\|u_{n}-u_{n-1}\right\|^{2} \\
\leq & \left\|u_{n-1}-u_{n}\right\|\left\{\left|1-\frac{r_{n}}{r_{n-1}}\right|\left\|u_{n-1}-x_{n-1}\right\|+\left\|x_{n-1}-x_{n}\right\|\right\}-\left\|u_{n}-u_{n-1}\right\|^{2},
\end{aligned}
$$

and then

$$
\left\|u_{n-1}-u_{n}\right\| \leq\left|1-\frac{r_{n}}{r_{n-1}}\right|\left\|u_{n-1}-x_{n-1}\right\|+\left\|x_{n-1}-x_{n}\right\| .
$$

Without loss of generality, let us assume that there exists a real number $\mu$ such that $r_{n}>$ $\mu>0$ for all positive integers $n$. Then we get

$$
\left\|u_{n-1}-u_{n}\right\| \leq\left\|x_{n-1}-x_{n}\right\|+\frac{1}{\mu}\left|r_{n-1}-r_{n}\right|\left\|u_{n-1}-x_{n-1}\right\|
$$

It follows from (3.7) and (3.12) that

$$
\begin{aligned}
\left\|y_{n}-y_{n-1}\right\| \leq & \beta_{n}\left\|x_{n}-x_{n-1}\right\|+\left(1-\beta_{n}\right)\left\{\left\|x_{n}-x_{n-1}\right\|+\frac{1}{\mu}\left|r_{n}-r_{n-1}\right|\left\|u_{n-1}-x_{n-1}\right\|\right. \\
& \left.+\left|\lambda_{n}-\lambda_{n-1}\right|\left\|A u_{n-1}\right\|\right\}+\left|\beta_{n}-\beta_{n-1}\right|\left(\left\|S x_{n-1}\right\|+\left\|z_{n-1}\right\|\right) \\
= & \left\|x_{n}-x_{n-1}\right\|+\left(1-\beta_{n}\right)\left\{\frac{1}{\mu}\left|r_{n}-r_{n-1}\right|\left\|u_{n-1}-x_{n-1}\right\|+\left|\lambda_{n}-\lambda_{n-1}\right|\left\|A u_{n-1}\right\|\right\} \\
& +\left|\beta_{n}-\beta_{n-1}\right|\left(\left\|S x_{n-1}\right\|+\left\|z_{n-1}\right\|\right) .
\end{aligned}
$$

Next, we estimate that

$$
\begin{aligned}
& \left\|x_{n+1}-x_{n}\right\| \\
& \quad \leq\left\|\alpha_{n} f\left(x_{n}\right)+\sum_{i=1}^{n}\left(\alpha_{i-1}-\alpha_{i}\right) V_{i} y_{n}-\left(\alpha_{n-1} f\left(x_{n-1}\right)+\sum_{i=1}^{n-1}\left(\alpha_{i-1}-\alpha_{i}\right) V_{i} y_{n-1}\right)\right\|
\end{aligned}
$$




$$
\begin{aligned}
= & \| \alpha_{n}\left(f\left(x_{n}\right)-f\left(x_{n-1}\right)\right)+\left(\alpha_{n}-\alpha_{n-1}\right) f\left(x_{n-1}\right)+\sum_{i=1}^{n}\left(\alpha_{i-1}-\alpha_{i}\right)\left(V_{i} y_{n}-V_{i} y_{n-1}\right) \\
& +\left(\alpha_{n-1}-\alpha_{n}\right) V_{n} y_{n-1} \| \\
\leq & \alpha_{n}\left\|f\left(x_{n}\right)-f\left(x_{n-1}\right)\right\|+\sum_{i=1}^{n}\left(\alpha_{i-1}-\alpha_{i}\right)\left\|V_{i} y_{n}-V_{i} y_{n-1}\right\| \\
& +\left|\alpha_{n}-\alpha_{n-1}\right|\left(\left\|f\left(x_{n-1}\right)\right\|+\left\|V_{n} y_{n-1}\right\|\right) \\
\leq & \alpha_{n} \rho\left\|x_{n}-x_{n-1}\right\|+\sum_{i=1}^{n}\left(\alpha_{i-1}-\alpha_{i}\right)\left\|y_{n}-y_{n-1}\right\| \\
& +\left|\alpha_{n}-\alpha_{n-1}\right|\left(\left\|f\left(x_{n-1}\right)\right\|+\left\|V_{n} y_{n-1}\right\|\right) \\
= & \alpha_{n} \rho\left\|x_{n}-x_{n-1}\right\|+\left(1-\alpha_{n}\right)\left\|y_{n}-y_{n-1}\right\| \\
& +\left|\alpha_{n}-\alpha_{n-1}\right|\left(\left\|f\left(x_{n-1}\right)\right\|+\left\|V_{n} y_{n-1}\right\|\right) .
\end{aligned}
$$

From (3.13) and (3.14), we have

$$
\begin{aligned}
\left\|x_{n+1}-x_{n}\right\| \leq & \alpha_{n} \rho\left\|x_{n}-x_{n-1}\right\| \\
& +\left(1-\alpha_{n}\right)\left\{\left\|x_{n}-x_{n-1}\right\|\right. \\
& +\left(1-\beta_{n}\right)\left(\frac{1}{\mu}\left|r_{n}-r_{n-1}\right|\left\|u_{n-1}-x_{n-1}\right\|+\left|\lambda_{n}-\lambda_{n-1}\right|\left\|A u_{n-1}\right\|\right) \\
& \left.+\left|\beta_{n}-\beta_{n-1}\right|\left(\left\|S x_{n-1}\right\|+\left\|z_{n-1}\right\|\right)\right\}+\left|\alpha_{n}-\alpha_{n-1}\right|\left(\left\|f\left(x_{n-1}\right)\right\|+\left\|V_{n} y_{n-1}\right\|\right) \\
\leq & \left(1-(1-\rho) \alpha_{n}\right)\left\|x_{n}-x_{n-1}\right\|+\frac{1}{\mu}\left|r_{n}-r_{n-1}\right|\left\|u_{n-1}-x_{n-1}\right\| \\
& +\left|\lambda_{n}-\lambda_{n-1}\right|\left\|A u_{n-1}\right\| \\
& +\left|\beta_{n}-\beta_{n-1}\right|\left(\left\|S x_{n-1}\right\|+\left\|z_{n-1}\right\|\right)+\left|\alpha_{n}-\alpha_{n-1}\right|\left(\left\|f\left(x_{n-1}\right)\right\|+\left\|V_{n} y_{n-1}\right\|\right) \\
\leq & \left(1-(1-\rho) \alpha_{n}\right)\left\|x_{n}-x_{n-1}\right\| \\
& +M\left(\frac{1}{\mu}\left|r_{n}-r_{n-1}\right|+\left|\lambda_{n}-\lambda_{n-1}\right|+\left|\beta_{n}-\beta_{n-1}\right|+\left|\alpha_{n}-\alpha_{n-1}\right|\right) .
\end{aligned}
$$

Where

$$
\begin{aligned}
M= & \max \left\{\sup _{n \geq 1}\left\|u_{n-1}-x_{n-1}\right\|, \sup _{n \geq 1}\left\|A u_{n-1}\right\|, \sup _{n \geq 1}\left(\left\|S x_{n-1}\right\|+\left\|z_{n-1}\right\|\right),\right. \\
& \left.\sup _{n \geq 1}\left(\left\|f\left(x_{n-1}\right)\right\|+\left\|V_{n} y_{n-1}\right\|\right)\right\} .
\end{aligned}
$$

It follows by conditions (a)-(e) of Algorithm 3.1 and Lemma 2.6 that

$$
\lim _{n \rightarrow \infty}\left\|x_{n+1}-x_{n}\right\|=0
$$


Next, we show that $\lim _{n \rightarrow \infty}\left\|u_{n}-x_{n}\right\|=0$. Since $x^{*} \in F(T) \cap \Omega^{*} \cap \operatorname{MEP}(F)$ and $\alpha_{n}+$ $\sum_{i=1}^{n}\left(\alpha_{i-1}-\alpha_{i}\right)=1$, by using (3.2) and (3.3), we obtain

$$
\begin{aligned}
\left\|x_{n+1}-x^{*}\right\|^{2} \leq & \left\|\alpha_{n} f\left(x_{n}\right)+\sum_{i=1}^{n}\left(\alpha_{i-1}-\alpha_{i}\right) V_{i} y_{n}-\alpha_{n} x^{*}-\sum_{i=1}^{n}\left(\alpha_{i-1}-\alpha_{i}\right) V_{i} x^{*}\right\|^{2} \\
\leq & \alpha_{n}\left\|f\left(x_{n}\right)-x^{*}\right\|^{2}+\sum_{i=1}^{n}\left(\alpha_{i-1}-\alpha_{i}\right)\left\|V_{i} y_{n}-V_{i} x^{*}\right\|^{2} \\
\leq & \alpha_{n}\left\|f\left(x_{n}\right)-x^{*}\right\|^{2}+\sum_{i=1}^{n}\left(\alpha_{i-1}-\alpha_{i}\right)\left\|y_{n}-x^{*}\right\|^{2} \\
\leq & \alpha_{n}\left\|f\left(x_{n}\right)-x^{*}\right\|^{2}+\left(1-\alpha_{n}\right)\left(\beta_{n}\left\|S x_{n}-x^{*}\right\|^{2}\right. \\
& \left.+\left(1-\beta_{n}\right)\left\|z_{n}-x^{*}\right\|^{2}\right) \\
\leq & \alpha_{n}\left\|f\left(x_{n}\right)-x^{*}\right\|^{2}+\left(1-\alpha_{n}\right) \beta_{n}\left\|S x_{n}-x^{*}\right\|^{2} \\
& +\left(1-\alpha_{n}\right)\left(1-\beta_{n}\right)\left\{\left\|x_{n}-x^{*}\right\|^{2}-r_{n}\left(2 \theta-r_{n}\right)\left\|D x_{n}-D x^{*}\right\|^{2}\right. \\
& \left.-\lambda_{n}\left(2 \alpha-\lambda_{n}\right)\left\|A u_{n}-A x^{*}\right\|^{2}\right\} \\
\leq & \alpha_{n}\left\|f\left(x_{n}\right)-x^{*}\right\|^{2}+\beta_{n}\left\|S x_{n}-x^{*}\right\|^{2}+\left\|x_{n}-x^{*}\right\|^{2} \\
& -\left(1-\alpha_{n}\right)\left(1-\beta_{n}\right)\left\{r_{n}\left(2 \theta-r_{n}\right)\left\|D x_{n}-D x^{*}\right\|^{2}\right. \\
& \left.+\lambda_{n}\left(2 \alpha-\lambda_{n}\right)\left\|A u_{n}-A x^{*}\right\|^{2}\right\} .
\end{aligned}
$$

Then from the inequality above, we get

$$
\begin{aligned}
& \left(1-\alpha_{n}\right)\left(1-\beta_{n}\right)\left\{r_{n}\left(2 \theta-r_{n}\right)\left\|D x_{n}-D x^{*}\right\|^{2}+\lambda_{n}\left(2 \alpha-\lambda_{n}\right)\left\|A u_{n}-A x^{*}\right\|^{2}\right\} \\
& \quad \leq \alpha_{n}\left\|f\left(x_{n}\right)-x^{*}\right\|^{2}+\beta_{n}\left\|S x_{n}-x^{*}\right\|^{2}+\left\|x_{n}-x^{*}\right\|^{2}-\left\|x_{n+1}-x^{*}\right\|^{2} \\
& \leq \alpha_{n}\left\|f\left(x_{n}\right)-x^{*}\right\|^{2}+\beta_{n}\left\|S x_{n}-x^{*}\right\|^{2}+\left(\left\|x_{n}-x^{*}\right\|+\left\|x_{n+1}-x^{*}\right\|\right)\left\|x_{n+1}-x_{n}\right\| .
\end{aligned}
$$

Since $\liminf _{n \rightarrow \infty} \lambda_{n} \leq \limsup \sup _{n \rightarrow \infty} \lambda_{n}<2 \alpha, \lim _{n \rightarrow \infty}\left\|x_{n+1}-x_{n}\right\|=0, \alpha_{n} \rightarrow 0$ and $\beta_{n} \rightarrow 0$, we obtain $\lim _{n \rightarrow \infty}\left\|D x_{n}-D x^{*}\right\|=0$ and $\lim _{n \rightarrow \infty}\left\|A u_{n}-A x^{*}\right\|=0$.

Since $T_{r_{n}}$ is firmly nonexpansive, we have

$$
\begin{aligned}
\left\|u_{n}-x^{*}\right\|^{2}= & \left\|T_{r_{n}}\left(x_{n}-r_{n} D x_{n}\right)-T_{r_{n}}\left(x^{*}-r_{n} D x^{*}\right)\right\|^{2} \\
\leq & \left\langle u_{n}-x^{*},\left(x_{n}-r_{n} D x_{n}\right)-\left(x^{*}-r_{n} D x^{*}\right)\right\rangle \\
= & \frac{1}{2}\left\{\left\|u_{n}-x^{*}\right\|^{2}+\left\|\left(x_{n}-r_{n} D x_{n}\right)-\left(x^{*}-r_{n} D x^{*}\right)\right\|^{2}\right. \\
& \left.-\left\|u_{n}-x^{*}-\left[\left(x_{n}-r_{n} D x_{n}\right)-\left(x^{*}-r_{n} D x^{*}\right)\right]\right\|^{2}\right\} .
\end{aligned}
$$

Hence,

$$
\begin{aligned}
\left\|u_{n}-x^{*}\right\|^{2} & \leq\left\|\left(x_{n}-r_{n} D x_{n}\right)-\left(x^{*}-r_{n} D x^{*}\right)\right\|^{2}-\left\|u_{n}-x_{n}+r_{n}\left(D x_{n}-D x^{*}\right)\right\|^{2} \\
& \leq\left\|x_{n}-x^{*}\right\|^{2}-\left\|u_{n}-x_{n}+r_{n}\left(D x_{n}-D x^{*}\right)\right\|^{2} \\
& \leq\left\|x_{n}-x^{*}\right\|^{2}-\left\|u_{n}-x_{n}\right\|^{2}+2 r_{n}\left\|u_{n}-x_{n}\right\|\left\|D x_{n}-D x^{*}\right\| .
\end{aligned}
$$


From (3.16), (3.3) and the inequality above, we have

$$
\begin{aligned}
\left\|x_{n+1}-x^{*}\right\|^{2} \leq & \alpha_{n}\left\|f\left(x_{n}\right)-x^{*}\right\|^{2}+\left(1-\alpha_{n}\right)\left(\beta_{n}\left\|S x_{n}-x^{*}\right\|^{2}+\left(1-\beta_{n}\right)\left\|z_{n}-x^{*}\right\|^{2}\right) \\
\leq & \alpha_{n}\left\|f\left(x_{n}\right)-x^{*}\right\|^{2}+\left(1-\alpha_{n}\right)\left(\beta_{n}\left\|S x_{n}-x^{*}\right\|^{2}+\left(1-\beta_{n}\right)\left\|u_{n}-x^{*}\right\|^{2}\right) \\
\leq & \alpha_{n}\left\|f\left(x_{n}\right)-x^{*}\right\|^{2}+\left(1-\alpha_{n}\right)\left\{\beta_{n}\left\|S x_{n}-x^{*}\right\|^{2}\right. \\
& \left.+\left(1-\beta_{n}\right)\left(\left\|x_{n}-x^{*}\right\|^{2}-\left\|u_{n}-x_{n}\right\|^{2}+2 r_{n}\left\|u_{n}-x_{n}\right\|\left\|D x_{n}-D x^{*}\right\|\right)\right\} \\
\leq & \alpha_{n}\left\|f\left(x_{n}\right)-x^{*}\right\|^{2}+\beta_{n}\left\|S x_{n}-x^{*}\right\|^{2}+\left\|x_{n}-x^{*}\right\|^{2} \\
& \quad-\left(1-\alpha_{n}\right)\left(1-\beta_{n}\right)\left\|u_{n}-x_{n}\right\|^{2}+2 r_{n}\left\|u_{n}-x_{n}\right\|\left\|D x_{n}-D x^{*}\right\| .
\end{aligned}
$$

Hence,

$$
\begin{aligned}
& \left(1-\alpha_{n}\right)\left(1-\beta_{n}\right)\left\|u_{n}-x_{n}\right\|^{2} \\
& \leq \alpha_{n}\left\|f\left(x_{n}\right)-x^{*}\right\|^{2}+\beta_{n}\left\|S x_{n}-x^{*}\right\|^{2}+\left\|x_{n}-x^{*}\right\|^{2}-\left\|x_{n+1}-x^{*}\right\|^{2} \\
& \quad+2 r_{n}\left\|u_{n}-x_{n}\right\|\left\|D x_{n}-D x^{*}\right\| \\
& \leq \alpha_{n}\left\|f\left(x_{n}\right)-x^{*}\right\|^{2}+\beta_{n}\left\|S x_{n}-x^{*}\right\|^{2}+\left(\left\|x_{n}-x^{*}\right\|+\left\|x_{n+1}-x^{*}\right\|\right)\left\|x_{n+1}-x_{n}\right\| \\
& \quad+2 r_{n}\left\|u_{n}-x_{n}\right\|\left\|D x_{n}-D x^{*}\right\| .
\end{aligned}
$$

Since $\lim _{n \rightarrow \infty}\left\|x_{n+1}-x_{n}\right\|=0, \alpha_{n} \rightarrow 0, \beta_{n} \rightarrow 0$ and $\lim _{n \rightarrow \infty}\left\|D x_{n}-D x^{*}\right\|=0$, we obtain

$$
\lim _{n \rightarrow \infty}\left\|u_{n}-x_{n}\right\|=0
$$

From (2.2), we get

$$
\begin{aligned}
\left\|z_{n}-x^{*}\right\|^{2}= & \left\|P_{C}\left[u_{n}-\lambda_{n} A u_{n}\right]-P_{C}\left[x^{*}-\lambda_{n} A x^{*}\right]\right\|^{2} \\
\leq & \left\langle z_{n}-x^{*},\left(u_{n}-\lambda_{n} A u_{n}\right)-\left(x^{*}-\lambda_{n} A x^{*}\right)\right\rangle \\
= & \frac{1}{2}\left\{\left\|z_{n}-x^{*}\right\|^{2}+\left\|u_{n}-x^{*}-\lambda_{n}\left(A u_{n}-A x^{*}\right)\right\|^{2}\right. \\
& \left.-\left\|u_{n}-x^{*}-\lambda_{n}\left(A u_{n}-A x^{*}\right)-\left(z_{n}-x^{*}\right)\right\|^{2}\right\} \\
\leq & \frac{1}{2}\left\{\left\|z_{n}-x^{*}\right\|^{2}+\left\|u_{n}-x^{*}\right\|^{2}-\left\|u_{n}-z_{n}-\lambda_{n}\left(A u_{n}-A x^{*}\right)\right\|^{2}\right\} \\
\leq & \frac{1}{2}\left\{\left\|z_{n}-x^{*}\right\|^{2}+\left\|u_{n}-x^{*}\right\|^{2}-\left\|u_{n}-z_{n}\right\|^{2}+2 \lambda_{n}\left\langle u_{n}-z_{n}, A u_{n}-A x^{*}\right\rangle\right\} \\
\leq & \frac{1}{2}\left\{\left\|z_{n}-x^{*}\right\|^{2}+\left\|u_{n}-x^{*}\right\|^{2}-\left\|u_{n}-z_{n}\right\|^{2}\right. \\
& \left.+2 \lambda_{n}\left\|u_{n}-z_{n}\right\|\left\|A u_{n}-A x^{*}\right\|\right\} .
\end{aligned}
$$

Hence,

$$
\begin{aligned}
\left\|z_{n}-x^{*}\right\|^{2} & \leq\left\|u_{n}-x^{*}\right\|^{2}-\left\|u_{n}-z_{n}\right\|^{2}+2 \lambda_{n}\left\|u_{n}-z_{n}\right\|\left\|A u_{n}-A x^{*}\right\| \\
& \leq\left\|x_{n}-x^{*}\right\|^{2}-\left\|u_{n}-z_{n}\right\|^{2}+2 \lambda_{n}\left\|u_{n}-z_{n}\right\|\left\|A u_{n}-A x^{*}\right\|
\end{aligned}
$$


From (3.16) and the inequality above, we have

$$
\begin{aligned}
\left\|x_{n+1}-x^{*}\right\|^{2} \leq & \alpha_{n}\left\|f\left(x_{n}\right)-x^{*}\right\|^{2}+\left(1-\alpha_{n}\right)\left(\beta_{n}\left\|S x_{n}-x^{*}\right\|^{2}+\left(1-\beta_{n}\right)\left\|z_{n}-x^{*}\right\|^{2}\right) \\
\leq & \alpha_{n}\left\|f\left(x_{n}\right)-x^{*}\right\|^{2}+\left(1-\alpha_{n}\right)\left\{\beta_{n}\left\|S x_{n}-x^{*}\right\|^{2}+\left(1-\beta_{n}\right)\left(\left\|x_{n}-x^{*}\right\|^{2}\right.\right. \\
& \left.\left.-\left\|u_{n}-z_{n}\right\|^{2}+2 \lambda_{n}\left\|u_{n}-z_{n}\right\|\left\|A u_{n}-A x^{*}\right\|\right)\right\} \\
\leq & \alpha_{n}\left\|f\left(x_{n}\right)-x^{*}\right\|^{2}+\beta_{n}\left\|S x_{n}-x^{*}\right\|^{2}+\left\|x_{n}-x^{*}\right\|^{2} \\
& -\left(1-\alpha_{n}\right)\left(1-\beta_{n}\right)\left\|u_{n}-z_{n}\right\|^{2}+2 \lambda_{n}\left\|u_{n}-z_{n}\right\|\left\|A u_{n}-A x^{*}\right\| .
\end{aligned}
$$

Hence,

$$
\begin{aligned}
& \left(1-\alpha_{n}\right)\left(1-\beta_{n}\right)\left\|u_{n}-z_{n}\right\|^{2} \\
& \leq \alpha_{n}\left\|f\left(x_{n}\right)-x^{*}\right\|^{2}+\beta_{n}\left\|S x_{n}-x^{*}\right\|^{2}+\left\|x_{n}-x^{*}\right\|^{2}-\left\|x_{n+1}-x^{*}\right\|^{2} \\
& \quad+2 \lambda_{n}\left\|u_{n}-z_{n}\right\|\left\|A u_{n}-A x^{*}\right\| \\
& \leq \alpha_{n}\left\|f\left(x_{n}\right)-x^{*}\right\|^{2}+\beta_{n}\left\|S x_{n}-x^{*}\right\|^{2}+\left(\left\|x_{n}-x^{*}\right\|+\left\|x_{n+1}-x^{*}\right\|\right)\left\|x_{n+1}-x_{n}\right\| \\
& \quad+2 \lambda_{n}\left\|u_{n}-z_{n}\right\|\left\|A u_{n}-A x^{*}\right\| .
\end{aligned}
$$

Since $\lim _{n \rightarrow \infty}\left\|x_{n+1}-x_{n}\right\|=0, \alpha_{n} \rightarrow 0, \beta_{n} \rightarrow 0$ and $\lim _{n \rightarrow \infty}\left\|A u_{n}-A x^{*}\right\|=0$, we obtain

$$
\lim _{n \rightarrow \infty}\left\|u_{n}-z_{n}\right\|=0
$$

It follows from (3.17) and (3.18) that

$$
\lim _{n \rightarrow \infty}\left\|x_{n}-z_{n}\right\|=0
$$

Now, let $z \in F(T) \cap \Omega^{*} \cap M E P(F)$, since for each $i \geq 1, V_{i} x_{n} \in C$ and $\alpha_{n}+\sum_{i=1}^{n}\left(\alpha_{i-1}-\alpha_{i}\right)=1$, we have $\sum_{i=1}^{n}\left(\alpha_{i-1}-\alpha_{i}\right) V_{i} x_{n}+\alpha_{n} z \in C$. And

$$
\begin{aligned}
\sum_{i=1}^{n}\left(\alpha_{i-1}-\alpha_{i}\right)\left(x_{n}-V_{i} x_{n}\right)= & P_{C}\left[\alpha_{n} f\left(x_{n}\right)+\sum_{i=1}^{n}\left(\alpha_{i-1}-\alpha_{i}\right) V_{i} y_{n}\right]+\left(1-\alpha_{n}\right) x_{n} \\
& -\left(\sum_{i=1}^{n}\left(\alpha_{i-1}-\alpha_{i}\right) V_{i} x_{n}+\alpha_{n} z\right)+\alpha_{n} z-x_{n+1} \\
= & P_{C}\left[\alpha_{n} f\left(x_{n}\right)+\sum_{i=1}^{n}\left(\alpha_{i-1}-\alpha_{i}\right) V_{i} y_{n}\right]+\alpha_{n}\left(z-x_{n+1}\right) \\
& -P_{C}\left[\sum_{i=1}^{n}\left(\alpha_{i-1}-\alpha_{i}\right) V_{i} x_{n}+\alpha_{n} z\right]+\left(1-\alpha_{n}\right)\left(x_{n}-x_{n+1}\right) .
\end{aligned}
$$

It follows that

$$
\begin{aligned}
& \sum_{i=1}^{n}\left(\alpha_{i-1}-\alpha_{i}\right)\left\langle x_{n}-V_{i} x_{n}, x_{n}-x^{*}\right\rangle \\
& \quad=\left\langle P_{C}\left[\alpha_{n} f\left(x_{n}\right)+\sum_{i=1}^{n}\left(\alpha_{i-1}-\alpha_{i}\right) V_{i} y_{n}\right]\right.
\end{aligned}
$$




$$
\begin{aligned}
& \left.-P_{C}\left[\sum_{i=1}^{n}\left(\alpha_{i-1}-\alpha_{i}\right) V_{i} x_{n}+\alpha_{n} z\right], x_{n}-x^{*}\right\rangle \\
& +\alpha_{n}\left\langle z-x_{n+1}, x_{n}-x^{*}\right\rangle+\left(1-\alpha_{n}\right)\left(x_{n}-x_{n+1}, x_{n}-x^{*}\right\rangle \\
\leq & \left\|\alpha_{n}\left(f\left(x_{n}\right)-z\right)+\sum_{i=1}^{n}\left(\alpha_{i-1}-\alpha_{i}\right)\left(V_{i} y_{n}-V_{i} x_{n}\right)\right\|\left\|x_{n}-x^{*}\right\| \\
& +\alpha_{n}\left\|z-x_{n+1}\right\|\left\|x_{n}-x^{*}\right\|+\left(1-\alpha_{n}\right)\left\|x_{n}-x_{n+1}\right\|\left\|x_{n}-x^{*}\right\| \\
\leq & \alpha_{n}\left\|f\left(x_{n}\right)-z\right\|\left\|x_{n}-x^{*}\right\|+\sum_{i=1}^{n}\left(\alpha_{i-1}-\alpha_{i}\right)\left\|y_{n}-x_{n}\right\|\left\|x_{n}-x^{*}\right\| \\
& +\alpha_{n}\left\|z-x_{n+1}\right\|\left\|x_{n}-x^{*}\right\|+\left(1-\alpha_{n}\right)\left\|x_{n}-x_{n+1}\right\|\left\|x_{n}-x^{*}\right\| \\
= & \alpha_{n}\left\|f\left(x_{n}\right)-z\right\|\left\|x_{n}-x^{*}\right\|+\left(1-\alpha_{n}\right)\left\|y_{n}-x_{n}\right\|\left\|x_{n}-x^{*}\right\| \\
& +\alpha_{n}\left\|z-x_{n+1}\right\|\left\|x_{n}-x^{*}\right\|+\left(1-\alpha_{n}\right)\left\|x_{n}-x_{n+1}\right\|\left\|x_{n}-x^{*}\right\| \\
\leq & \alpha_{n}\left\|f\left(x_{n}\right)-z\right\|\left\|x_{n}-x^{*}\right\|+\left(1-\alpha_{n}\right)\left\|\beta_{n} S x_{n}+\left(1-\beta_{n}\right) z_{n}-x_{n}\right\|\left\|x_{n}-x^{*}\right\| \\
& +\alpha_{n}\left\|z-x_{n+1}\right\|\left\|x_{n}-x^{*}\right\|+\left(1-\alpha_{n}\right)\left\|x_{n}-x_{n+1}\right\|\left\|x_{n}-x^{*}\right\| \\
\leq & \alpha_{n}\left\|f\left(x_{n}\right)-z\right\|\left\|x_{n}-x^{*}\right\|+\left(1-\alpha_{n}\right) \beta_{n}\left\|S x_{n}-x_{n}\right\|\left\|x_{n}-x^{*}\right\| \\
& +\left(1-\alpha_{n}\right)\left(1-\beta_{n}\right)\left\|z_{n}-x_{n}\right\|\left\|x_{n}-x^{*}\right\| \\
& +\alpha_{n}\left\|z-x_{n+1}\right\|\left\|x_{n}-x^{*}\right\|+\left(1-\alpha_{n}\right)\left\|x_{n}-x_{n+1}\right\|\left\|x_{n}-x^{*}\right\| .
\end{aligned}
$$

From Lemma 2.9 and the inequality above, we get

$$
\begin{aligned}
& \frac{1}{2} \sum_{i=1}^{n}\left(\alpha_{i-1}-\alpha_{i}\right)\left\|x_{n}-V_{i} x_{n}\right\|^{2} \\
& \quad \leq \sum_{i=1}^{n}\left(\alpha_{i-1}-\alpha_{i}\right)\left\langle x_{n}-V_{i} x_{n}, x_{n}-x^{*}\right\rangle \\
& \leq \alpha_{n}\left\|f\left(x_{n}\right)-z\right\|\left\|x_{n}-x^{*}\right\|+\left(1-\alpha_{n}\right) \beta_{n}\left\|S x_{n}-x_{n}\right\|\left\|x_{n}-x^{*}\right\| \\
& \quad+\left(1-\alpha_{n}\right)\left(1-\beta_{n}\right)\left\|z_{n}-x_{n}\right\|\left\|x_{n}-x^{*}\right\|+\alpha_{n}\left\|z-x_{n+1}\right\|\left\|x_{n}-x^{*}\right\| \\
& \quad+\left(1-\alpha_{n}\right)\left\|x_{n}-x_{n+1}\right\|\left\|x_{n}-x^{*}\right\| .
\end{aligned}
$$

Since $\lim _{n \rightarrow \infty}\left\|x_{n+1}-x_{n}\right\|=0, \alpha_{n} \rightarrow 0, \beta_{n} \rightarrow 0$ and $\lim _{n \rightarrow \infty}\left\|x_{n}-z_{n}\right\|=0$, we obtain

$$
\lim _{n \rightarrow \infty} \sum_{i=1}^{n}\left(\alpha_{i-1}-\alpha_{i}\right)\left\|x_{n}-V_{i} x_{n}\right\|^{2}=0
$$

Since $\left(\alpha_{i-1}-\alpha_{i}\right)\left\|x_{n}-V_{i} x_{n}\right\|^{2} \leq \sum_{i=1}^{n}\left(\alpha_{i-1}-\alpha_{i}\right)\left\|x_{n}-V_{i} x_{n}\right\|^{2}$ and $\left\{\alpha_{n}\right\}$ is strictly decreasing, we have

$$
\lim _{n \rightarrow \infty}\left\|x_{n}-V_{i} x_{n}\right\|=0 .
$$


Hence, we obtain

$$
\lim _{n \rightarrow \infty}\left\|x_{n}-T_{i} x_{n}\right\|=\lim _{n \rightarrow \infty} \frac{\left\|x_{n}-V_{i} x_{n}\right\|}{\left(1-k_{i}\right)}=0, \quad \forall i \geq 1
$$

Since $\left\{x_{n}\right\}$ is bounded, without loss of generality, we can assume that $x_{n} \rightarrow w \in C$. It follows from Lemma 2.4 that $w \in F(T)$. Therefore, $w_{w}\left(x_{n}\right) \subset F(T)$.

Theorem 3.1 The sequence $\left\{x_{n}\right\}$ generated by Algorithm 3.1 converges strongly to $z=$ $P_{\Omega^{*} \cap M E P(F) \cap F(T)} f(z)$, which is the unique solution of the variational inequality

$$
\langle(I-f) z, x-z\rangle \geq 0, \quad \forall x \in \Omega^{*} \cap M E P(F) \cap F(T) .
$$

Proof Since $\left\{x_{n}\right\}$ is bounded $x_{n} \rightarrow w$ and from Lemma 3.2, we have $w \in F(T)$. Next, we show that $w \in \operatorname{MEP}(F)$. Since $u_{n}=T_{r_{n}}\left(x_{n}-r_{n} D x_{n}\right)$, we have

$$
F\left(u_{n}, y\right)+\left\langle D x_{n}, y-u_{n}\right\rangle+\frac{1}{r_{n}}\left\langle y-u_{n}, u_{n}-x_{n}\right\rangle \geq 0, \quad \forall y \in C .
$$

It follows from monotonicity of $F$ that

$$
\left\langle D x_{n}, y-u_{n}\right\rangle+\frac{1}{r_{n}}\left\langle y-u_{n}, u_{n}-x_{n}\right\rangle \geq F\left(y, u_{n}\right), \quad \forall y \in C
$$

and

$$
\left\langle D x_{n_{k}}, y-u_{n_{k}}\right\rangle+\left\langle y-u_{n_{k}}, \frac{u_{n_{k}}-x_{n_{k}}}{r_{n_{k}}}\right\rangle \geq F\left(y, u_{n_{k}}\right), \quad \forall y \in C .
$$

Since $\lim _{n \rightarrow \infty}\left\|u_{n}-x_{n}\right\|=0$ and $x_{n} \rightarrow w$, it easy to observe that $u_{n_{k}} \rightarrow w$. For any $0<t \leq 1$ and $y \in C$, let $y_{t}=t y+(1-t) w$, we have $y_{t} \in C$. Then from (3.21), we obtain

$$
\begin{aligned}
\left\langle D y_{t}, y_{t}-u_{n_{k}}\right\rangle \geq & \left\langle D y_{t}, y_{t}-u_{n_{k}}\right\rangle-\left\langle D x_{n_{k}}, y_{t}-u_{n_{k}}\right\rangle-\left\langle y_{t}-u_{n_{k}}, \frac{u_{n_{k}}-x_{n_{k}}}{r_{n_{k}}}\right\rangle+F\left(y_{t}, u_{n_{k}}\right) \\
= & \left\langle D y_{t}-D u_{n_{k}}, y_{t}-u_{n_{k}}\right\rangle+\left\langle D u_{n_{k}}-D x_{n_{k}}, y_{t}-u_{n_{k}}\right\rangle \\
& -\left\langle y_{t}-u_{n_{k}}, \frac{u_{n_{k}}-x_{n_{k}}}{r_{n_{k}}}\right\rangle+F\left(y_{t}, u_{n_{k}}\right) .
\end{aligned}
$$

Since $D$ is Lipschitz continuous and $\lim _{n \rightarrow \infty}\left\|u_{n}-x_{n}\right\|=0$, we obtain $\lim _{k \rightarrow \infty} \| D u_{n_{k}}-$ $D x_{n_{k}} \|=0$. From the monotonicity of $D$ and $u_{n_{k}} \rightarrow w$, it follows from (3.22) that

$$
\left\langle D y_{t}, y_{t}-w\right\rangle \geq F\left(y_{t}, w\right) \text {. }
$$

Hence, from assumptions (i)-(iv) of Lemma 2.2 and (3.23), we have

$$
\begin{aligned}
0=F\left(y_{t}, y_{t}\right) & \leq t F\left(y_{t}, y\right)+(1-t) F\left(y_{t}, w\right) \\
& \leq t F\left(y_{t}, y\right)+(1-t)\left\langle D y_{t}, y_{t}-w\right\rangle \\
& \leq t F\left(y_{t}, y\right)+(1-t) t\left\langle D y_{t}, y-w\right\rangle,
\end{aligned}
$$


which implies that $F\left(y_{t}, y\right)+(1-t)\left\langle D y_{t}, y-w\right\rangle \geq 0$. Letting $t \rightarrow 0_{+}$, we have

$$
F(w, y)+\langle D w, y-w\rangle \geq 0, \quad \forall y \in C,
$$

which implies that $w \in M E P(F)$.

Furthermore, we show that $w \in \Omega^{*}$. Let

$$
T v= \begin{cases}A v+N_{C} v, & \forall v \in C, \\ \emptyset, & \text { otherwise }\end{cases}
$$

where $N_{C} v:=\{w \in H:\langle w, v-u\rangle \geq 0, \forall u \in C\}$ is the normal cone to $C$ at $v \in C$. Then $T$ is maximal monotone and $0 \in T v$ if and only if $v \in \Omega^{*}$ (see [18]). Let $G(T)$ denote the graph of $T$, and let $(v, u) \in G(T)$, since $u-A v \in N_{C} v$ and $z_{n} \in C$, we have

$$
\left\langle v-z_{n}, u-A v\right\rangle \geq 0
$$

On the other hand, it follows from $z_{n}=P_{C}\left[u_{n}-\lambda_{n} A u_{n}\right]$ and $v \in C$ that

$$
\left\langle v-z_{n}, z_{n}-\left(u_{n}-\lambda_{n} A u_{n}\right)\right\rangle \geq 0
$$

and

$$
\left\langle v-z_{n}, \frac{z_{n}-u_{n}}{\lambda_{n}}+A u_{n}\right\rangle \geq 0
$$

Therefore, from (3.25) and inverse strongly monotonicity of $A$, we have

$$
\begin{aligned}
\left\langle v-z_{n_{k}}, u\right\rangle & \geq\left\langle v-z_{n_{k}}, A v\right\rangle \\
& \geq\left\langle v-z_{n_{k}}, A v\right\rangle-\left\langle v-z_{n_{k}}, \frac{z_{n_{k}}-u_{n_{k}}}{\lambda_{n_{k}}}+A u_{n_{k}}\right\rangle \\
& \geq\left\langle v-z_{n_{k}}, A v-A z_{n_{k}}\right\rangle+\left\langle v-z_{n_{k}}, A z_{n_{k}}-A u_{n_{k}}\right\rangle-\left\langle v-z_{n_{k}}, \frac{z_{n_{k}}-u_{n_{k}}}{\lambda_{n_{k}}}\right\rangle \\
& \geq\left\langle v-z_{n_{k}}, A z_{n_{k}}-A u_{n_{k}}\right\rangle-\left\langle v-z_{n_{k}}, \frac{z_{n_{k}}-u_{n_{k}}}{\lambda_{n_{k}}}\right\rangle .
\end{aligned}
$$

Since $\lim _{n \rightarrow \infty}\left\|u_{n}-z_{n}\right\|=0$ and $u_{n_{k}} \rightarrow w$, it easy to observe that $z_{n_{k}} \rightarrow w$. Hence, we obtain $\langle v-w, u\rangle \geq 0$. Since $T$ is maximal monotone, we have $w \in T^{-1} 0$, and hence, $w \in \Omega^{*}$. Thus, we have

$$
w \in \Omega^{*} \cap M E P(F) \cap F(T) .
$$

Next, we claim that $\lim _{\sup _{n \rightarrow \infty}}\left\langle f(z)-z, x_{n}-z\right\rangle \leq 0$, where $z=P_{\Omega^{*} \cap M E P(F) \cap F(T)} f(z)$.

Since $\left\{x_{n}\right\}$ is bounded, there exists a subsequence $\left\{x_{n_{k}}\right\}$ of $\left\{x_{n}\right\}$ such that

$$
\limsup _{n \rightarrow \infty}\left\langle f(z)-z, x_{n}-z\right\rangle=\limsup _{k \rightarrow \infty}\left\langle f(z)-z, x_{n_{k}}-z\right\rangle=\langle f(z)-z, w-z\rangle \leq 0 .
$$


Next, we show that $x_{n} \rightarrow z$.

$$
\begin{aligned}
& \left\|x_{n+1}-z\right\|^{2}=\left\langle x_{n+1}-\alpha_{n} f\left(x_{n}\right)-\sum_{i=1}^{n}\left(\alpha_{i-1}-\alpha_{i}\right) V_{i} y_{n}, x_{n+1}-z\right\rangle \\
& +\left\langle\alpha_{n} f\left(x_{n}\right)+\sum_{i=1}^{n}\left(\alpha_{i-1}-\alpha_{i}\right) V_{i} y_{n}-z, x_{n+1}-z\right\rangle \\
& \leq\left\langle\alpha_{n} f\left(x_{n}\right)+\sum_{i=1}^{n}\left(\alpha_{i-1}-\alpha_{i}\right) V_{i} y_{n}-z, x_{n+1}-z\right\rangle \\
& \leq \alpha_{n}\left\langle f\left(x_{n}\right)-f(z), x_{n+1}-z\right\rangle+\alpha_{n}\left\langle f(z)-z, x_{n+1}-z\right\rangle \\
& +\sum_{i=1}^{n}\left(\alpha_{i-1}-\alpha_{i}\right)\left\langle V_{i} y_{n}-z, x_{n+1}-z\right\rangle \\
& \leq \alpha_{n}\left\|f\left(x_{n}\right)-f(z)\right\|\left\|x_{n+1}-z\right\|+\alpha_{n}\left\langle f(z)-z, x_{n+1}-z\right\rangle \\
& +\sum_{i=1}^{n}\left(\alpha_{i-1}-\alpha_{i}\right)\left\|V_{i} y_{n}-z\right\|\left\|x_{n+1}-z\right\| \\
& \leq \alpha_{n} \rho\left\|x_{n}-z\right\|\left\|x_{n+1}-z\right\|+\alpha_{n}\left\langle f(z)-z, x_{n+1}-z\right\rangle \\
& +\sum_{i=1}^{n}\left(\alpha_{i-1}-\alpha_{i}\right)\left\|y_{n}-z\right\|\left\|x_{n+1}-z\right\| \\
& \leq \alpha_{n} \rho\left\|x_{n}-z\right\|\left\|x_{n+1}-z\right\|+\alpha_{n}\left\langle f(z)-z, x_{n+1}-z\right\rangle \\
& +\left(1-\alpha_{n}\right)\left\{\beta_{n}\left\|S x_{n}-S z\right\|+\beta_{n}\|S z-z\|+\left(1-\beta_{n}\right)\left\|z_{n}-z\right\|\right\}\left\|x_{n+1}-z\right\| \\
& \leq \alpha_{n} \rho\left\|x_{n}-z\right\|\left\|x_{n+1}-z\right\|+\alpha_{n}\left\langle f(z)-z, x_{n+1}-z\right\rangle \\
& +\left(1-\alpha_{n}\right)\left\{\beta_{n}\left\|x_{n}-z\right\|+\beta_{n}\|S z-z\|+\left(1-\beta_{n}\right)\left\|x_{n}-z\right\|\right\}\left\|x_{n+1}-z\right\| \\
& \leq\left(1-\alpha_{n}(1-\rho)\right)\left\|x_{n}-z\right\|\left\|x_{n+1}-z\right\|+\alpha_{n}\left\langle f(z)-z, x_{n+1}-z\right\rangle \\
& +\left(1-\alpha_{n}\right) \beta_{n}\|S z-z\|\left\|x_{n+1}-z\right\| \\
& \leq \frac{1-\alpha_{n}(1-\rho)}{2}\left(\left\|x_{n}-z\right\|^{2}+\left\|x_{n+1}-z\right\|^{2}\right)+\alpha_{n}\left\langle f(z)-z, x_{n+1}-z\right\rangle \\
& +\left(1-\alpha_{n}\right) \beta_{n}\|S z-z\|\left\|x_{n+1}-z\right\| \text {, }
\end{aligned}
$$

which implies that

$$
\begin{aligned}
\left\|x_{n+1}-z\right\|^{2} \leq & \left(1-\frac{2 \alpha_{n}(1-\rho)}{1+\alpha_{n}(1-\rho)}\right)\left\|x_{n}-z\right\|^{2}+\frac{2 \alpha_{n}}{1+\alpha_{n}(1-\rho)}\left\langle f(z)-z, x_{n+1}-z\right\rangle \\
& +\frac{2\left(1-\alpha_{n}\right) \beta_{n}}{1+\alpha_{n}(1-\rho)}\|S z-z\|\left\|x_{n+1}-z\right\| \\
\leq & \left(1-\frac{2 \alpha_{n}(1-\rho)}{1+\alpha_{n}(1-\rho)}\right)\left\|x_{n}-z\right\|^{2}+\frac{2 \alpha_{n}(1-\rho)}{1+\alpha_{n}(1-\rho)}\left\{\frac{1}{1-\rho}\left\langle f(z)-z, x_{n+1}-z\right\rangle\right. \\
& \left.+\frac{\left(1-\alpha_{n}\right) \beta_{n}}{\alpha_{n}(1-\rho)}\|S z-z\|\left\|x_{n+1}-z\right\|\right\} .
\end{aligned}
$$

Let $\gamma_{n}=\frac{2 \alpha_{n}(1-\rho)}{1+\alpha_{n}(1-\rho)}$ and $\delta_{n}=\frac{2 \alpha_{n}(1-\rho)}{1+\alpha_{n}(1-\rho)}\left\{\frac{1}{1-\rho}\left\langle f(z)-z, x_{n+1}-z\right\rangle+\frac{\left(1-\alpha_{n}\right) \beta_{n}}{\alpha_{n}(1-\rho)}\|S z-z\|\left\|x_{n+1}-z\right\|\right\}$. 
Since

$$
\begin{aligned}
& \sum_{n=1}^{\infty} \alpha_{n}=\infty, \quad 1+\alpha_{n}(1-\rho) \leq 2 \text { and } \\
& \limsup _{n \rightarrow \infty}\left\{\frac{1}{1-\rho}\left\langle f(z)-z, x_{n+1}-z\right\rangle+\frac{\left(1-\alpha_{n}\right) \beta_{n}}{\alpha_{n}(1-\rho)}\|S z-z\|\left\|x_{n+1}-z\right\|\right\} \leq 0 .
\end{aligned}
$$

It follows that

$$
\sum_{n=1}^{\infty} \gamma_{n}=\infty \quad \text { and } \quad \limsup _{n \rightarrow \infty} \frac{\delta_{n}}{\gamma_{n}} \leq 0
$$

Thus, all the conditions of Lemma 2.6 are satisfied. Hence, we deduce that $x_{n} \rightarrow z$.

Since $P_{\Omega^{*} \cap M E P(F) \cap F(T)} f$ is a contraction, there exists a unique $z \in C$ such that $z=$ $P_{\Omega^{*} \cap M E P(F) \cap F(T)} f(z)$. From (2.1), it follows that $z$ is the unique solution of problem (3.20). This completes the proof.

Theorem 3.2 Let $C$ be a nonempty closed convex subset of a real Hilbert space H. Let $D, A: C \rightarrow H$ be $\theta, \alpha$-inverse strongly monotone mappings, respectively. Let $F: C \times C \rightarrow \mathbb{R}$ be a bifunction satisfying the assumptions (i)-(iv) of Lemma 2.2, $S: C \rightarrow H$ be a nonexpansive mapping, and $\left\{T_{i}\right\}_{i=1}^{\infty}: C \rightarrow C$ is a countable family of $k_{i}$-strict pseudo-contraction mappings such that $F(T) \cap \Omega^{*} \cap \operatorname{MEP}(F) \neq \emptyset$, where $F(T)=\bigcap_{i=1}^{\infty} F\left(T_{i}\right)$. Let $f$ be a $\rho$ contraction mapping. For a given $x_{0} \in C$ arbitrarily, let the iterative sequences $\left\{u_{n}\right\},\left\{x_{n}\right\}$, $\left\{y_{n}\right\}$ and $\left\{z_{n}\right\}$ be generated by

$$
\begin{aligned}
& F\left(u_{n}, y\right)+\left\langle D x_{n}, y-u_{n}\right\rangle+\frac{1}{r_{n}}\left\langle y-u_{n}, u_{n}-x_{n}\right\rangle \geq 0, \quad \forall y \in C ; \\
& z_{n}=P_{C}\left[u_{n}-\lambda_{n} A u_{n}\right] ; \\
& y_{n}=\beta_{n} S x_{n}+\left(1-\beta_{n}\right) z_{n} ; \\
& x_{n+1}=P_{C}\left[\alpha_{n} f\left(x_{n}\right)+\sum_{i=1}^{n}\left(\alpha_{i-1}-\alpha_{i}\right) V_{i} y_{n}\right], \quad \forall n \geq 0,
\end{aligned}
$$

where $V_{i}=k_{i} I+\left(1-k_{i}\right) T_{i}, 0 \leq k_{i}<1, \alpha_{0}=1,\left\{\alpha_{n}\right\}$ is a strictly decreasing sequence in $(0,1)$, and $\left\{\beta_{n}\right\}$ is a sequence in $(0,1)$ satisfying the following conditions:

(a) $\lim _{n \rightarrow \infty} \alpha_{n}=0$ and $\sum_{n=1}^{\infty} \alpha_{n}=\infty$,

(b) $\lim _{n \rightarrow \infty} \frac{\beta_{n}}{\alpha_{n}}=\tau \in(0, \infty)$,

(c) $\sum_{n=1}^{\infty}\left(\alpha_{n-1}-\alpha_{n}\right)<\infty$ and $\sum_{n=1}^{\infty}\left|\beta_{n-1}-\beta_{n}\right|<\infty$,

(d) $\lim _{n \rightarrow \infty} \frac{\frac{1}{\mu}\left|r_{n}-r_{n-1}\right|+\left|\lambda_{n}-\lambda_{n-1}\right|+\left|\alpha_{n-1}-\alpha_{n}\right|+\left|\beta_{n-1}-\beta_{n}\right|}{\alpha_{n} \beta_{n}}=0$,

(e) there exists a constant $K>0$ such that $\frac{1}{\alpha_{n}}\left|\frac{1}{\beta_{n}}-\frac{1}{\beta_{n-1}}\right| \leq K$,

(f) $\liminf _{n \rightarrow \infty} r_{n}>0$ and $\sum_{n=1}^{\infty}\left|r_{n-1}-r_{n}\right|<\infty$,

(g) $\liminf _{n \rightarrow \infty} \lambda_{n}<\limsup _{n \rightarrow \infty} \lambda_{n}<2 \alpha$ and $\sum_{n=1}^{\infty}\left|\lambda_{n-1}-\lambda_{n}\right|<\infty$.

Then sequence $\left\{x_{n}\right\}$ generated by (3.26) converges strongly to $x^{*} \in \Omega^{*} \cap \operatorname{MEP}(F) \cap F(T)$, which is the unique solution of the variational inequality

$$
\left\langle\frac{1}{\tau}(I-f) x^{*}+(I-S) x^{*}, x-x^{*}\right\rangle \geq 0, \quad \forall x \in \Omega^{*} \cap M E P(F) \cap F(T) .
$$


Proof From $\lim _{n \rightarrow \infty}\left(\beta_{n} / \alpha_{n}\right)=\tau \in(0, \infty)$, without loss of generality, we can assume that $\beta_{n} \leq(1+\tau) \alpha_{n}$ for all $n \geq 1$. Hence, $\beta_{n} \rightarrow 0$. By similar argument as that in Lemmas 3.1 and 3.2, we can deduce that $\left\{x_{n}\right\}$ is bounded, $\lim _{n \rightarrow \infty}\left\|x_{n+1}-x_{n}\right\|=0, \lim _{n \rightarrow \infty}\left\|x_{n}-z_{n}\right\|=0$ (see (3.19)) and $\left(I-V_{i}\right) x_{n} \rightarrow 0$. Then we have

$$
\left\|y_{n}-x_{n}\right\| \leq \beta_{n}\left\|x_{n}-S x_{n}\right\|+\left(1-\beta_{n}\right)\left\|x_{n}-z_{n}\right\| \rightarrow 0 \quad \text { as } n \rightarrow \infty
$$

It follows that for all $i \geq 1$,

$$
\left\|y_{n}-V_{i} x_{n}\right\| \leq\left\|y_{n}-x_{n}\right\|+\left\|x_{n}-V_{i} x_{n}\right\| \rightarrow 0 \quad \text { as } n \rightarrow \infty
$$

From (3.28) and (3.29), we have

$$
\begin{aligned}
\left\|y_{n}-V_{i} y_{n}\right\| & \leq\left\|y_{n}-V_{i} x_{n}\right\|+\left\|V_{i} x_{n}-V_{i} y_{n}\right\| \\
& \leq\left\|y_{n}-V_{i} x_{n}\right\|+\left\|y_{n}-x_{n}\right\| \rightarrow 0 \quad \text { as } n \rightarrow \infty .
\end{aligned}
$$

Set $w_{n}=\alpha_{n} f\left(x_{n}\right)+\sum_{i=1}^{n}\left(\alpha_{i-1}-\alpha_{i}\right) V_{i} y_{n}$. From (3.14) and (3.15), we obtain

$$
\begin{aligned}
& \frac{\left\|x_{n+1}-x_{n}\right\|}{\beta_{n}} \leq \frac{\left\|w_{n}-w_{n-1}\right\|}{\beta_{n}} \\
& \leq\left(1-(1-\rho) \alpha_{n}\right) \frac{\left\|x_{n}-x_{n-1}\right\|}{\beta_{n}} \\
& +M\left(\frac{1}{\mu} \frac{\left|r_{n}-r_{n-1}\right|}{\beta_{n}}+\frac{\left|\lambda_{n}-\lambda_{n-1}\right|}{\beta_{n}}+\frac{\left|\beta_{n}-\beta_{n-1}\right|}{\beta_{n}}+\frac{\left|\alpha_{n}-\alpha_{n-1}\right|}{\beta_{n}}\right) \\
& =\left(1-(1-\rho) \alpha_{n}\right) \frac{\left\|x_{n}-x_{n-1}\right\|}{\beta_{n-1}}+\left(1-(1-\rho) \alpha_{n}\right)\left\|x_{n}-x_{n-1}\right\|\left(\frac{1}{\beta_{n}}-\frac{1}{\beta_{n-1}}\right) \\
& +M\left(\frac{1}{\mu} \frac{\left|r_{n}-r_{n-1}\right|}{\beta_{n}}+\frac{\left|\lambda_{n}-\lambda_{n-1}\right|}{\beta_{n}}+\frac{\left|\beta_{n}-\beta_{n-1}\right|}{\beta_{n}}+\frac{\left|\alpha_{n}-\alpha_{n-1}\right|}{\beta_{n}}\right) \\
& \leq\left(1-(1-\rho) \alpha_{n}\right) \frac{\left\|x_{n}-x_{n-1}\right\|}{\beta_{n-1}}+\left\|x_{n}-x_{n-1}\right\|\left|\frac{1}{\beta_{n}}-\frac{1}{\beta_{n-1}}\right| \\
& +M\left(\frac{1}{\mu} \frac{\left|r_{n}-r_{n-1}\right|}{\beta_{n}}+\frac{\left|\lambda_{n}-\lambda_{n-1}\right|}{\beta_{n}}+\frac{\left|\beta_{n}-\beta_{n-1}\right|}{\beta_{n}}+\frac{\left|\alpha_{n}-\alpha_{n-1}\right|}{\beta_{n}}\right) \\
& \leq\left(1-(1-\rho) \alpha_{n}\right) \frac{\left\|x_{n}-x_{n-1}\right\|}{\beta_{n-1}}+\alpha_{n} K\left\|x_{n}-x_{n-1}\right\| \\
& +M\left(\frac{1}{\mu} \frac{\left|r_{n}-r_{n-1}\right|}{\beta_{n}}+\frac{\left|\lambda_{n}-\lambda_{n-1}\right|}{\beta_{n}}+\frac{\left|\beta_{n}-\beta_{n-1}\right|}{\beta_{n}}+\frac{\left|\alpha_{n}-\alpha_{n-1}\right|}{\beta_{n}}\right) \\
& \leq\left(1-(1-\rho) \alpha_{n}\right) \frac{\left\|w_{n-1}-w_{n-2}\right\|}{\beta_{n-1}}+\alpha_{n} K\left\|x_{n}-x_{n-1}\right\| \\
& +M\left(\frac{1}{\mu} \frac{\left|r_{n}-r_{n-1}\right|}{\beta_{n}}+\frac{\left|\lambda_{n}-\lambda_{n-1}\right|}{\beta_{n}}+\frac{\left|\beta_{n}-\beta_{n-1}\right|}{\beta_{n}}+\frac{\left|\alpha_{n}-\alpha_{n-1}\right|}{\beta_{n}}\right) .
\end{aligned}
$$

Let $\gamma_{n}=(1-\rho) \alpha_{n}$ and $\delta_{n}=\alpha_{n} K\left\|x_{n}-x_{n-1}\right\|+M\left(\frac{1}{\mu} \frac{\left|r_{n}-r_{n-1}\right|}{\beta_{n}}+\frac{\left|\lambda_{n}-\lambda_{n-1}\right|}{\beta_{n}}+\frac{\left|\beta_{n}-\beta_{n-1}\right|}{\beta_{n}}+\frac{\left|\alpha_{n}-\alpha_{n-1}\right|}{\beta_{n}}\right)$. From conditions (a) and (d) of Theorem 3.2, we have

$$
\sum_{n=1}^{\infty} \gamma_{n}=\infty \quad \text { and } \quad \lim _{n \rightarrow \infty} \frac{\delta_{n}}{\gamma_{n}}=0
$$


By Lemma 2.6, we obtain

$$
\lim _{n \rightarrow \infty} \frac{\left\|x_{n+1}-x_{n}\right\|}{\beta_{n}}=0, \quad \lim _{n \rightarrow \infty} \frac{\left\|w_{n+1}-w_{n}\right\|}{\beta_{n}}=\lim _{n \rightarrow \infty} \frac{\left\|w_{n+1}-w_{n}\right\|}{\alpha_{n}}=0 .
$$

From (3.26), we have

$$
x_{n+1}=P_{C}\left[w_{n}\right]-w_{n}+\alpha_{n} f\left(x_{n}\right)+\sum_{i=1}^{n}\left(\alpha_{i-1}-\alpha_{i}\right)\left(V_{i} y_{n}-y_{n}\right)+\left(1-\alpha_{n}\right) y_{n} .
$$

Hence, it follows that

$$
\begin{aligned}
x_{n}-x_{n+1}= & \left(1-\alpha_{n}\right) x_{n}+\alpha_{n} x_{n} \\
& -\left(P_{C}\left[w_{n}\right]-w_{n}+\alpha_{n} f\left(x_{n}\right)+\sum_{i=1}^{n}\left(\alpha_{i-1}-\alpha_{i}\right)\left(V_{i} y_{n}-y_{n}\right)+\left(1-\alpha_{n}\right) y_{n}\right) \\
= & \left(1-\alpha_{n}\right)\left[\beta_{n}\left(x_{n}-S x_{n}\right)+\left(1-\beta_{n}\right)\left(x_{n}-z_{n}\right)\right]+\left(w_{n}-P_{C}\left[w_{n}\right]\right) \\
& +\sum_{i=1}^{n}\left(\alpha_{i-1}-\alpha_{i}\right)\left(y_{n}-V_{i} y_{n}\right)+\alpha_{n}\left(x_{n}-f\left(x_{n}\right)\right),
\end{aligned}
$$

and hence,

$$
\begin{aligned}
\frac{x_{n}-x_{n+1}}{\left(1-\alpha_{n}\right) \beta_{n}}= & x_{n}-S x_{n}+\frac{\left(1-\beta_{n}\right)}{\beta_{n}}\left(x_{n}-z_{n}\right)+\frac{1}{\left(1-\alpha_{n}\right) \beta_{n}}\left(w_{n}-P_{C}\left[w_{n}\right]\right) \\
& +\frac{1}{\left(1-\alpha_{n}\right) \beta_{n}} \sum_{i=1}^{n}\left(\alpha_{i-1}-\alpha_{i}\right)\left(y_{n}-V_{i} y_{n}\right)+\frac{\alpha_{n}}{\left(1-\alpha_{n}\right) \beta_{n}}\left(x_{n}-f\left(x_{n}\right)\right) .
\end{aligned}
$$

Let $v_{n}=\frac{x_{n}-x_{n+1}}{\left(1-\alpha_{n}\right) \beta_{n}}$. For any $z \in \Omega^{*} \cap M E P(F) \cap F(T)$, we have

$$
\begin{aligned}
\left\langle v_{n}, x_{n}-z\right\rangle= & \frac{1}{\left(1-\alpha_{n}\right) \beta_{n}}\left\langle w_{n}-P_{C}\left[w_{n}\right], P_{C}\left[w_{n-1}\right]-z\right\rangle+\frac{\alpha_{n}}{\left(1-\alpha_{n}\right) \beta_{n}}\left\langle(I-f) x_{n}, x_{n}-z\right\rangle \\
& +\left\langle x_{n}-S x_{n}, x_{n}-z\right\rangle+\frac{\left(1-\beta_{n}\right)}{\beta_{n}}\left\langle x_{n}-z_{n}, x_{n}-z\right\rangle \\
& +\frac{1}{\left(1-\alpha_{n}\right) \beta_{n}} \sum_{i=1}^{n}\left(\alpha_{i-1}-\alpha_{i}\right)\left\langle y_{n}-V_{i} y_{n}, x_{n}-z\right\rangle .
\end{aligned}
$$

Since $S$ is a nonexpansive mapping, $f$ is a $\rho$-contraction mapping, and $V_{i}$ is a $k_{i}$-strict pseudo-contraction mapping. Then $(I-S)$ and $\left(I-V_{i}\right)$ are monotones, and $f$ is strongly monotone with coefficient $(1-\rho)$. We can deduce

$$
\begin{aligned}
\left\langle x_{n}-S x_{n}, x_{n}-z\right\rangle & =\left\langle(I-S) x_{n}-(I-S) z, x_{n}-z\right\rangle+\left\langle(I-S) z, x_{n}-z\right\rangle \\
& \geq\left\langle(I-S) z, x_{n}-z\right\rangle, \\
\left\langle(I-f) x_{n}, x_{n}-z\right\rangle & =\left\langle(I-f) x_{n}-(I-f) z, x_{n}-z\right\rangle+\left\langle(I-f) z, x_{n}-z\right\rangle \\
& \geq(1-\rho)\left\|x_{n}-z\right\|^{2}+\left\langle(I-f) z, x_{n}-z\right\rangle,
\end{aligned}
$$




$$
\begin{aligned}
\left\langle\left(I-V_{i}\right) y_{n}, x_{n}-z\right\rangle= & \left\langle\left(I-V_{i}\right) y_{n}-\left(I-V_{i}\right) z, x_{n}-y_{n}\right\rangle \\
& +\left\langle\left(I-V_{i}\right) y_{n}-\left(I-V_{i}\right) z, y_{n}-z\right\rangle \\
\geq & \left\langle\left(I-V_{i}\right) y_{n}-\left(I-V_{i}\right) z, x_{n}-y_{n}\right\rangle \\
= & \left\langle\left(I-V_{i}\right) y_{n}, x_{n}-y_{n}\right\rangle \\
= & \left\langle\left(I-V_{i}\right) y_{n}, \beta_{n}\left(x_{n}-S x_{n}\right)+\left(1-\beta_{n}\right)\left(x_{n}-z_{n}\right)\right\rangle .
\end{aligned}
$$

From (2.1), we get

$$
\begin{aligned}
\left\langle w_{n}\right. & \left.-P_{C}\left[w_{n}\right], P_{C}\left[w_{n-1}\right]-z\right\rangle \\
& =\left\langle w_{n}-P_{C}\left[w_{n}\right], P_{C}\left[w_{n-1}\right]-P_{C}\left[w_{n}\right]\right\rangle+\left\langle w_{n}-P_{C}\left[w_{n}\right], P_{C}\left[w_{n}\right]-z\right\rangle \\
& \geq\left\langle w_{n}-P_{C}\left[w_{n}\right], P_{C}\left[w_{n-1}\right]-P_{C}\left[w_{n}\right]\right\rangle .
\end{aligned}
$$

Then from (3.30)-(3.32), we have

$$
\begin{aligned}
\left\langle v_{n}, x_{n}-z\right\rangle \geq & \frac{1}{\left(1-\alpha_{n}\right) \beta_{n}}\left\langle w_{n}-P_{C}\left[w_{n}\right], P_{C}\left[w_{n-1}\right]-P_{C}\left[w_{n}\right]\right\rangle+\frac{\alpha_{n}}{\left(1-\alpha_{n}\right) \beta_{n}}\left\langle(I-f) z, x_{n}-z\right\rangle \\
& +\left\langle(I-S) z, x_{n}-z\right\rangle+\frac{\left(1-\beta_{n}\right)}{\beta_{n}}\left\langle x_{n}-z_{n}, x_{n}-z\right\rangle \\
& +\frac{\left(1-\beta_{n}\right)}{\left(1-\alpha_{n}\right) \beta_{n}} \sum_{i=1}^{n}\left(\alpha_{i-1}-\alpha_{i}\right)\left\langle\left(I-V_{i}\right) y_{n}, x_{n}-z_{n}\right\rangle \\
& +\frac{1}{\left(1-\alpha_{n}\right)} \sum_{i=1}^{n}\left(\alpha_{i-1}-\alpha_{i}\right)\left\langle\left(I-V_{i}\right) y_{n}, x_{n}-S x_{n}\right\rangle+\frac{(1-\rho) \alpha_{n}}{\left(1-\alpha_{n}\right) \beta_{n}}\left\|x_{n}-z\right\|^{2} .
\end{aligned}
$$

Then we obtain

$$
\begin{aligned}
\left\|x_{n}-z\right\|^{2} \leq & \frac{1}{(1-\rho) \alpha_{n}}\left\|w_{n}-P_{C}\left[w_{n}\right]\right\|\left\|w_{n-1}-w_{n}\right\|-\frac{1}{(1-\rho)}\left\langle(I-f) z, x_{n}-z\right\rangle \\
& +\frac{\left(1-\alpha_{n}\right) \beta_{n}}{(1-\rho) \alpha_{n}}\left(\left\langle v_{n}, x_{n}-z\right\rangle-\left\langle(I-S) z, x_{n}-z\right\rangle\right) \\
& -\frac{\left(1-\beta_{n}\right)\left(1-\alpha_{n}\right)}{(1-\rho) \alpha_{n}}\left\langle x_{n}-z_{n}, x_{n}-z\right\rangle \\
& -\frac{\left(1-\beta_{n}\right)}{(1-\rho) \alpha_{n}} \sum_{i=1}^{n}\left(\alpha_{i-1}-\alpha_{i}\right)\left\langle\left(I-V_{i}\right) y_{n}, x_{n}-z_{n}\right\rangle \\
& -\frac{\beta_{n}}{(1-\rho) \alpha_{n}} \sum_{i=1}^{n}\left(\alpha_{i-1}-\alpha_{i}\right)\left\langle\left(I-V_{i}\right) y_{n}, x_{n}-S x_{n}\right\rangle . \\
\leq & \frac{\left\|w_{n-1}-w_{n}\right\|}{(1-\rho) \alpha_{n}}\left\|w_{n}-P_{C}\left[w_{n}\right]\right\|-\frac{1}{(1-\rho)}\left\langle(I-f) z, x_{n}-z\right\rangle \\
& +\frac{\left(1-\alpha_{n}\right) \beta_{n}}{(1-\rho) \alpha_{n}}\left(\left\langle v_{n}, x_{n}-z\right\rangle-\left\langle(I-S) z, x_{n}-z\right\rangle\right) \\
& +\frac{1}{(1-\rho)} \frac{\left(1-\beta_{n}\right)}{\beta_{n}} \frac{\beta_{n}}{\alpha_{n}}\left\|x_{n}-z_{n}\right\|\left\|x_{n}-z\right\|
\end{aligned}
$$




$$
\begin{aligned}
& +\frac{1}{(1-\rho)} \frac{\left(1-\beta_{n}\right)}{\beta_{n}} \frac{\beta_{n}}{\alpha_{n}} \sum_{i=1}^{n}\left(\alpha_{i-1}-\alpha_{i}\right)\left\|\left(I-V_{i}\right) y_{n}\right\|\left\|x_{n}-z_{n}\right\| \\
& -\frac{\beta_{n}}{(1-\rho) \alpha_{n}} \sum_{i=1}^{n}\left(\alpha_{i-1}-\alpha_{i}\right)\left\langle\left(I-V_{i}\right) y_{n}, x_{n}-S x_{n}\right\rangle .
\end{aligned}
$$

By condition (e) of Theorem 3.2, there exists a constant $N>0$ such that $\frac{1-\beta_{n}}{\beta_{n}} \leq N$. Since $\lim _{n \rightarrow \infty}\left\|x_{n}-z_{n}\right\|=0, v_{n} \rightarrow 0,\left(I-V_{i}\right) y_{n} \rightarrow 0$ and $\frac{\left\|w_{n-1}-w_{n}\right\|}{\alpha_{n}} \rightarrow 0$ as $n \rightarrow \infty$, then every weak cluster point of $\left\{x_{n}\right\}$ is also a strong cluster point. Since $\left\{x_{n}\right\}$ is bounded, by Lemma 3.2, there exists a subsequence $\left\{x_{n_{k}}\right\}$ of $\left\{x_{n}\right\}$ converging to a point $x^{*} \in F(T)$, by a similar argument as that in Theorem 3.1, we can show that $x^{*} \in \Omega^{*} \cap M E P(F) \cap F(T)$.

From (3.30)-(3.32), it follows that for any $z \in \Omega^{*} \cap M E P(F) \cap F(T)$,

$$
\begin{aligned}
& \left\langle(I-f) x_{n_{k}}, x_{n_{k}}-z\right\rangle \\
& =\frac{\left(1-\alpha_{n_{k}}\right) \beta_{n_{k}}}{\alpha_{n_{k}}}\left\langle v_{n_{k}}, x_{n_{k}}-z\right\rangle-\frac{1}{\alpha_{n_{k}}}\left\langle w_{n_{k}}-P_{C}\left[w_{n_{k}}\right], P_{C}\left[w_{n_{k}-1}\right]-z\right\rangle \\
& -\frac{\left(1-\alpha_{n_{k}}\right) \beta_{n_{k}}}{\alpha_{n_{k}}}\left\langle x_{n_{k}}-S x_{n_{k}}, x_{n_{k}}-z\right\rangle-\frac{\left(1-\alpha_{n_{k}}\right)\left(1-\beta_{n_{k}}\right)}{\alpha_{n_{k}}}\left\langle x_{n_{k}}-z_{n_{k}}, x_{n_{k}}-z\right\rangle \\
& -\frac{1}{\alpha_{n_{k}}} \sum_{i=1}^{n}\left(\alpha_{i-1}-\alpha_{i}\right)\left\langle y_{n_{k}}-V_{i} y_{n_{k}}, x_{n_{k}}-z\right\rangle \\
& \leq \frac{\left(1-\alpha_{n_{k}}\right) \beta_{n_{k}}}{\alpha_{n_{k}}}\left\langle v_{n_{k}}, x_{n_{k}}-z\right\rangle+\frac{1}{\alpha_{n_{k}}}\left\|w_{n_{k}}-P_{C}\left[w_{n_{k}}\right]\right\|\left\|w_{n_{k}-1}-w_{n_{k}}\right\| \\
& -\frac{\left(1-\alpha_{n_{k}}\right) \beta_{n_{k}}}{\alpha_{n_{k}}}\left\langle x_{n_{k}}-S x_{n_{k}}, x_{n_{k}}-z\right\rangle+\frac{\left(1-\beta_{n_{k}}\right)}{\beta_{n_{k}}} \frac{\beta_{n_{k}}}{\alpha_{n_{k}}}\left\|x_{n_{k}}-z_{n_{k}}\right\|\left\|x_{n_{k}}-z\right\| \\
& +\frac{\left(1-\beta_{n_{k}}\right)}{\beta_{n_{k}}} \frac{\beta_{n_{k}}}{\alpha_{n_{k}}} \sum_{i=1}^{n_{k}}\left(\alpha_{i-1}-\alpha_{i}\right)\left\|\left(I-V_{i}\right) y_{n_{k}}\right\|\left\|x_{n_{k}}-z_{n_{k}}\right\| \\
& -\frac{\beta_{n_{k}}}{\alpha_{n_{k}}} \sum_{i=1}^{n_{k}}\left(\alpha_{i-1}-\alpha_{i}\right)\left\langle\left(I-V_{i}\right) y_{n_{k}}, x_{n_{k}}-S x_{n_{k}}\right\rangle .
\end{aligned}
$$

Since $\lim _{n \rightarrow \infty}\left\|x_{n}-z_{n}\right\|=0, v_{n} \rightarrow 0,\left(I-V_{i}\right) y_{n} \rightarrow 0$ and $\frac{\left\|w_{n-1}-w_{n}\right\|}{\alpha_{n}} \rightarrow 0$, letting $k \rightarrow \infty$ in (3.34), we obtain

$$
\left\langle(I-f) x^{*}, x^{*}-z\right\rangle \leq-\tau\left\langle x^{*}-S x^{*}, x^{*}-z\right\rangle
$$

i.e.,

$$
\left\langle\frac{1}{\tau}(I-f) x^{*}+(I-S) x^{*}, z-x^{*}\right\rangle \geq 0 .
$$

In the following, we show that (3.27) has a unique solution. Assume that $x^{\prime}$ is another solution. Then we have

$$
\begin{aligned}
& \left\langle(I-f) x^{\prime}, x^{\prime}-x^{*}\right\rangle \leq-\tau\left\langle x^{\prime}-S x^{\prime}, x^{\prime}-x^{*}\right\rangle, \\
& \left\langle(I-f) x^{*}, x^{*}-x^{\prime}\right\rangle \leq-\tau\left\langle x^{*}-S x^{*}, x^{*}-x^{\prime}\right\rangle .
\end{aligned}
$$


Adding (3.35) and (3.36), we get

$$
\begin{aligned}
(1-\rho)\left\|x^{\prime}-x^{*}\right\|^{2} & \leq\left\langle(I-f) x^{\prime}-(I-f) x^{*}, x^{\prime}-x^{*}\right\rangle \\
& \leq-\tau\left\langle(I-S) x^{\prime}-(I-S) x^{*}, x^{\prime}-x^{*}\right\rangle \\
& \leq 0 .
\end{aligned}
$$

Then $x^{\prime}=x^{*}$. Since (3.27) has a unique solution, it follows that $w_{w}\left(x_{n}\right)=\left\{x^{*}\right\}$. Since every weak cluster point of $\left\{x_{n}\right\}$ is also a strong cluster point, we conclude that $\left\{x_{n}\right\} \rightarrow x^{*}$. This completes the proof.

\section{Applications}

In this section, we obtain the following results by using a special case of the proposed method. The first result can be viewed as extension and improvement of the method of Gu et al. [6] for finding the approximate element of the common set of solutions of a generalized equilibrium problem and a hierarchical fixed point problem in a real Hilbert space.

Corollary 4.1 Let $C$ be a nonempty closed convex subset of a real Hilbert space H. Let $D: C \rightarrow H$ be $\theta$-inverse strongly monotone mappings, respectively. Let $F: C \times C \rightarrow \mathbb{R}$ be a bifunction satisfying the assumptions (i)-(iv) of Lemma 2.2, $S: C \rightarrow H$ be a nonexpansive mapping, and $\left\{T_{i}\right\}_{i=1}^{\infty}: C \rightarrow C$ is a countable family of $k_{i}$-strict pseudo-contraction mappings such that $F(T) \cap M E P(F) \neq \emptyset$, where $F(T)=\bigcap_{i=1}^{\infty} F\left(T_{i}\right)$. Let $f$ be a $\rho$-contraction mapping. For a given $x_{0} \in C$ arbitrarily, let the iterative sequences $\left\{u_{n}\right\},\left\{x_{n}\right\},\left\{y_{n}\right\}$ and $\left\{z_{n}\right\}$ be generated by

$$
\begin{aligned}
& F\left(u_{n}, y\right)+\left\langle D x_{n}, y-u_{n}\right\rangle+\frac{1}{r_{n}}\left\langle y-u_{n}, u_{n}-x_{n}\right\rangle \geq 0, \quad \forall y \in C ; \\
& y_{n}=\beta_{n} S x_{n}+\left(1-\beta_{n}\right) u_{n} ; \\
& x_{n+1}=P_{C}\left[\alpha_{n} f\left(x_{n}\right)+\sum_{i=1}^{n}\left(\alpha_{i-1}-\alpha_{i}\right) T_{i} y_{n}\right], \quad \forall n \geq 0,
\end{aligned}
$$

where $\alpha_{0}=1,\left\{\alpha_{n}\right\}$ is a strictly decreasing sequence in $(0,1)$, and $\left\{\beta_{n}\right\}$ is a sequence in $(0,1)$ satisfying the following conditions:

(a) $\lim _{n \rightarrow \infty} \alpha_{n}=0$ and $\sum_{n=1}^{\infty} \alpha_{n}=\infty$,

(b) $\lim _{n \rightarrow \infty} \frac{\beta_{n}}{\alpha_{n}}=\tau \in(0, \infty)$,

(c) $\sum_{n=1}^{\infty}\left(\alpha_{n-1}-\alpha_{n}\right)<\infty$ and $\sum_{n=1}^{\infty}\left|\beta_{n-1}-\beta_{n}\right|<\infty$,

(d) $\lim _{n \rightarrow \infty} \frac{\frac{1}{\mu}\left|r_{n}-r_{n-1}\right|+\left|\alpha_{n-1}-\alpha_{n}\right|+\left|\beta_{n-1}-\beta_{n}\right|}{\alpha_{n} \beta_{n}}=0$,

(e) there exists a constant $K>0$ such that $\frac{1}{\alpha_{n}}\left|\frac{1}{\beta_{n}}-\frac{1}{\beta_{n-1}}\right| \leq K$,

(f) $\liminf _{n \rightarrow \infty} r_{n}>0$ and $\sum_{n=1}^{\infty}\left|r_{n-1}-r_{n}\right|<\infty$.

Then sequence $\left\{x_{n}\right\}$ generated by algorithm (4.1) converges strongly to $x^{*} \in M E P(F) \cap F(T)$, which is the unique solution of the variational inequality

$$
\left\langle\frac{1}{\tau}(I-f) x^{*}+(I-S) x^{*}, x-x^{*}\right\rangle \geq 0, \quad \forall x \in M E P(F) \cap F(T) .
$$


Proof Putting $A=0$ and $k_{i}=0, \forall i \geq 1$ in Theorem 3.2. Then conclusion of Corollary 4.1 is obtained.

The following result can be viewed as extension and improvement of the method of Yao et al. [20] for finding the approximate element of the common set of solutions of a generalized equilibrium problem and a hierarchical fixed point problem in a real Hilbert space.

Corollary 4.2 Let $C$ be a nonempty closed convex subset of a real Hilbert space H. Let $D: C \rightarrow H$ be $\theta$-inverse strongly monotone mappings, respectively. Let $F: C \times C \rightarrow \mathbb{R}$ be a bifunction satisfying assumptions (i)-(iv) of Lemma 2.2, $S: C \rightarrow H$ be a nonexpansive mapping, and $T: C \rightarrow C$ is a countable family of $k$-strict pseudo-contraction mappings such that $F(T) \cap M E P(F) \neq \emptyset$. Let $f$ be a $\rho$-contraction mapping. For a given $x_{0} \in C$ arbitrarily, let the iterative sequences $\left\{u_{n}\right\},\left\{x_{n}\right\},\left\{y_{n}\right\}$ and $\left\{z_{n}\right\}$ be generated by

$$
\begin{aligned}
& F\left(u_{n}, y\right)+\left\langle D x_{n}, y-u_{n}\right\rangle+\frac{1}{r_{n}}\left\langle y-u_{n}, u_{n}-x_{n}\right\rangle \geq 0, \quad \forall y \in C ; \\
& y_{n}=\beta_{n} S x_{n}+\left(1-\beta_{n}\right) u_{n} ; \\
& x_{n+1}=P_{C}\left[\alpha_{n} f\left(x_{n}\right)+\left(1-\alpha_{n}\right) T y_{n}\right], \quad \forall n \geq 0,
\end{aligned}
$$

where $\alpha_{0}=1,\left\{\alpha_{n}\right\}$ is a strictly decreasing sequence in $(0,1)$, and $\left\{\beta_{n}\right\}$ is a sequence in $(0,1)$ satisfying the following conditions:

(a) $\lim _{n \rightarrow \infty} \alpha_{n}=0$ and $\sum_{n=1}^{\infty} \alpha_{n}=\infty$,

(b) $\lim _{n \rightarrow \infty} \frac{\beta_{n}}{\alpha_{n}}=\tau \in(0, \infty)$,

(c) $\sum_{n=1}^{\infty}\left(\alpha_{n-1}-\alpha_{n}\right)<\infty$ and $\sum_{n=1}^{\infty}\left|\beta_{n-1}-\beta_{n}\right|<\infty$,

(d) $\lim _{n \rightarrow \infty} \frac{\frac{1}{\mu}\left|r_{n}-r_{n-1}\right|+\left|\alpha_{n-1}-\alpha_{n}\right|+\left|\beta_{n-1}-\beta_{n}\right|}{\alpha_{n} \beta_{n}}=0$,

(e) there exists a constant $K>0$ such that $\frac{1}{\alpha_{n}}\left|\frac{1}{\beta_{n}}-\frac{1}{\beta_{n-1}}\right| \leq K$,

(f) $\liminf _{n \rightarrow \infty} r_{n}>0$ and $\sum_{n=1}^{\infty}\left|r_{n-1}-r_{n}\right|<\infty$.

Then sequence $\left\{x_{n}\right\}$ generated by algorithm (4.2) converges strongly to $x^{*} \in \operatorname{MEP}(F) \cap F(T)$, which is the unique solution of the variational inequality

$$
\left\langle\frac{1}{\tau}(I-f) x^{*}+(I-S) x^{*}, x-x^{*}\right\rangle \geq 0, \quad \forall x \in M E P(F) \cap F(T) .
$$

Proof Putting $A=0, k_{i}=0$ and $T_{i}=T \forall i \geq 1$ in Theorem 3.2. Then conclusion of Corollary 4.2 is obtained.

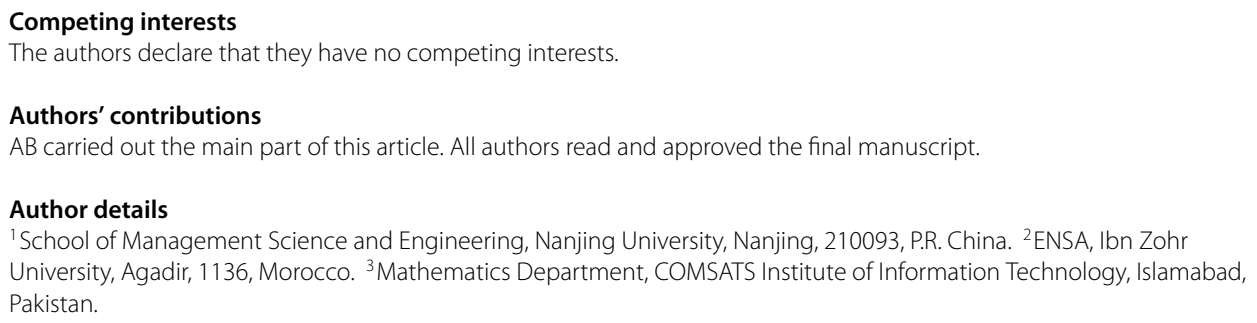




\section{References}

1. Acedo, GL, Xu, HK: Iterative methods for strictly pseudo-contractions in Hilbert space. Nonlinear Anal. 67, 2258-2271 (2007)

2. Blum, E, Oettli, W: From optimization and variational inequalities to equilibrium problems. Math. Stud. 63, 123-145 (1994)

3. Cianciaruso, F, Marino, G, Muglia, L, Yao, Y: On a two-steps algorithm for hierarchical fixed point problems and variational inequalities. J. Inequal. Appl. 2009, 1-13 (2009)

4. Chang, SS, Joseph Lee, HW, Chan, CK: A new method for solving equilibrium problem fixed point problem and variational inequality problem with application to optimization. Nonlinear Anal. 70, 3307-3319 (2009)

5. Combettes, PL, Hirstoaga, SA: Equilibrium programming using proximal like algorithms. Math. Program. 78, 29-41 (1997)

6. Gu, G, Wang, S, Cho, YJ: Strong convergence algorithms for hierarchical fixed points problems and variational inequalities. J. Appl. Math. 2011, 1-17 (2011)

7. Katchang, P, Kumam, P: A new iterative algorithm for equilibrium problems, variational inequalities and fixed point problems in a Hilbert space. Appl. Math. Comput. 32, 19-38 (2010)

8. Lions, JL, Stampacchia, G: Variational inequalities. Commun. Pure Appl. Math. 20, 493-512 (1967)

9. Mainge, PE, Moudafi, A: Strong convergence of an iterative method for hierarchical fixed-point problems. Pac. J. Optim. 3(3), 529-538 (2007)

10. Marino, G, Xu, HK: Convergence of generalized proximal point algorithms. Commun. Pure Appl. Anal. 3, 791-808 (2004)

11. Marino, G, Xu, HK: A general iterative method for nonexpansive mappings in Hilbert spaces. J. Math. Anal. Appl. 318(1), 43-52 (2006)

12. Marino, G, Xu, HK: Explicit hierarchical fixed point approach to variational inequalities. J. Optim. Theory Appl. 149(1), 61-78 (2011)

13. Moudafi, A, Théra, M: Proximal and Dynamical Approaches to Equilibrium Problems. Lecture Notes in Economics and Mathematical Systems, vol. 477. Springer, New York (1999)

14. Moudafi, A: Mixed equilibrium problems sensitivity analysis and algorithmic aspect. Comput. Math. Appl. 44, 1099-1108 (2002)

15. Moudafi, A: Krasnoselski-Mann iteration for hierarchical fixed-point problems. Inverse Probl. 23(4), 1635-1640 (2007)

16. Plubtieng, S, Punpaeng, R: A general iterative method for equilibrium problems and fixed point problems in Hilbert spaces. J. Math. Anal. Appl. 336, 455-469 (2007)

17. Qin, $X$, Shang, M, Su, Y: A general iterative method for equilibrium problem and fixed point problem in Hilbert spaces. Nonlinear Anal. 69, 3897-3909 (2008)

18. Rockafellar, RT: On the maximality of sums nonlinear monotone operators. Trans. Am. Math. Soc. 149, 75-88 (1970)

19. Xu, HK: Iterative algorithms for nonlinear operators. J. Lond. Math. Soc. 66, 240-256 (2002)

20. Yao, Y, Cho, YJ, Liou, YC: Iterative algorithms for hierarchical fixed points problems and variational inequalities. Math. Comput. Model. 52(9-10), 1697-1705 (2010)

21. Yao, Y, Liou, YC, Kang, SM: Approach to common elements of variational inequality problems and fixed point problems via a relaxed extragradient method. Comput. Math. Appl. 59(11), 3472-3480 (2010)

22. Zhou, $\mathrm{H}$ : Convergence theorems of fixed points for $\mathrm{k}$-strict pseudo-contractions in Hilbert spaces. Nonlinear Anal. 69 456-462 (2008)

10.1186/1029-242X-2013-490

Cite this article as: Bnouhachem and Noor: An iterative method for approximating the common solutions of a variational inequality, a mixed equilibrium problem and a hierarchical fixed point problem. Journal of Inequalities and Applications 2013, 2013:490

\section{Submit your manuscript to a SpringerOpen ${ }^{0}$ journal and benefit from:}

- Convenient online submission

- Rigorous peer review

Immediate publication on acceptance

- Open access: articles freely available online

- High visibility within the field

- Retaining the copyright to your article 